\title{
SEMICLASSICAL WAVE PACKET DYNAMICS FOR HARTREE EQUATIONS
}

\author{
PEI CAO AND RÉMI CARLES
}

\begin{abstract}
We study the propagation of wave packets for nonlinear nonlocal Schrödinger equations in the semi-classical limit. When the kernel is smooth, we construct approximate solutions for the wave functions in subcritical, critical and supercritical cases (in terms of the size of the initial data). The validity of the approximation is proved up to Ehrenfest time. For homogeneous kernels, we establish similar results in subcritical and critical cases. Nonlinear superposition principle for two nonlinear wave packets is also considered.
\end{abstract}

\section{INTRODUCTION}

In this paper, we consider the following semi-classically scaled Hartree equation

$$
i \varepsilon \partial_{t} \psi^{\varepsilon}+\frac{\varepsilon^{2}}{2} \Delta \psi^{\varepsilon}=V(t, x) \psi^{\varepsilon}+\left(K *\left|\psi^{\varepsilon}\right|^{2}\right) \psi^{\varepsilon}, \quad(t, x) \in \mathbf{R}_{+} \times \mathbf{R}^{d},
$$

where $K: \mathbf{R}^{d} \rightarrow \mathbf{R}, V: \mathbf{R}_{+} \times \mathbf{R}^{d} \rightarrow \mathbf{R}, d \geqslant 1$, with initial data

$$
\psi^{\varepsilon}(0, x)=\varepsilon^{M} \times \varepsilon^{-d / 4} a\left(\frac{x-x_{0}}{\sqrt{\varepsilon}}\right) e^{i\left(x-x_{0}\right) \cdot \xi_{0} / \varepsilon}, \quad a \in \mathcal{S}\left(\mathbf{R}^{d}\right), \quad x_{0}, \xi_{0} \in \mathbf{R}^{d} .
$$

Such data, which are called semi-classical wave packets (or coherent states), have raised great interest in the linear case (see e.g. [3, 8, 9, 20, 21]). It is well known that if the data is a wave packet, then the solution of the linear equation $(K=0)$ associated with (1.1) and (1.2) still is a wave packet at leading order up to times of order $C \log \left(\frac{1}{\varepsilon}\right)$, called Ehrenfest time (see e.g. [2, 16, 17]). We refer the reader to the recent papers $[8,22,23,24$, where overview and references on the topics can be found. Throughout this paper, we consider dynamical properties for positive time only: this is just for the simplicity of notations, since the equation is reversible.

This paper is inspired by the two recent papers [1], where (1.1) is considered for a smooth kernel $K$, and [6] where the nonlinearity is local, as opposed to the Hartree nonlinearity. In [6], the authors proved that if the initial data have subcritical size, the leading order behavior of the wave function as $\varepsilon \rightarrow 0$ is the same as for the linear equation. When the size of the initial data is critical, at leading order the wave function propagates like a coherent state whose envelope is given by a nonlinear equation, up to a nonlinear analogue of the Ehrenfest time. In this paper, we follow a similar approach in the case of nonlocal Schrödinger equations, a case where this analysis is not a priori clear, precisely because the nonlinearity is nonlocal.

This work was supported by the French ANR project R.A.S. (ANR-08-JCJC-0124-01), and was achieved when the first author was visiting the University of Montpellier 2, under a grant from Tsinghua University. She would like to thank these institutions for this opportunity. 
Up to changing $\psi^{\varepsilon}$ to $\varepsilon^{-M} \psi^{\varepsilon}$, (1.1) and (1.2) can be written as:

$$
\left\{\begin{aligned}
i \varepsilon \partial_{t} \psi^{\varepsilon}+\frac{\varepsilon^{2}}{2} \Delta \psi^{\varepsilon} & =V(t, x) \psi^{\varepsilon}+\varepsilon^{\alpha}\left(K *\left|\psi^{\varepsilon}\right|^{2}\right) \psi^{\varepsilon}, \\
\psi^{\varepsilon}(0, x) & =\varepsilon^{-d / 4} a\left(\frac{x-x_{0}}{\sqrt{\varepsilon}}\right) e^{i\left(x-x_{0}\right) \cdot \xi_{0} / \varepsilon},
\end{aligned}\right.
$$

with $\alpha=2 M$. Notice that the initial data are of order $\mathcal{O}(1)$ in $L^{2}\left(\mathbf{R}^{d}\right)$, and $\alpha$ accounts for the strength of nonlinear effects in the limit $\varepsilon \rightarrow 0$.

Consider the trajectories associated with the Hamiltonian flow $\frac{|\xi|^{2}}{2}+V(t, x(t))$ :

$$
\dot{x}(t)=\xi(t), \quad \dot{\xi}(t)=-\nabla V(t, x(t)) ; \quad x(0)=x_{0}, \xi(0)=\xi_{0} .
$$

Assumption 1.1. The external potential $V$ is smooth, real-valued, and at most quadratic in space:

$$
V \in C^{\infty}\left(\mathbf{R}_{+} \times \mathbf{R}^{d} ; \mathbf{R}\right), \quad \text { and } \quad \partial_{x}^{\beta} V \in L^{\infty}\left(\mathbf{R}_{+} \times \mathbf{R}^{d}\right), \quad \forall|\beta| \geqslant 2 .
$$

In addition, we require $t \mapsto \nabla V(t, 0) \in L^{\infty}\left(\mathbf{R}_{+}\right)$.

Remark 1.2. If $V=V(x)$ does not depend on time, the last assumption is automatically fulfilled. This assumption is needed to ensure that the Hamiltonian flow grows at most exponentially in time. Typically, if $V(x)=\kappa \cdot x e^{e^{t}}$ for some (constant) $\kappa \in \mathbf{R}^{d}$, then $\dot{\xi}(t)=-\kappa e^{e^{t}}$, so $x$ and $\xi$ grow like a double exponential.

The following lemma is straightforward.

Lemma 1.3. Let $\left(x_{0}, \xi_{0}\right) \in \mathbf{R}^{d} \times \mathbf{R}^{d}$. Under Assumption 1.1, (1.4) has a unique global, smooth solution $(x, \xi) \in C^{\infty}\left(\mathbf{R}_{+} ; \mathbf{R}^{d}\right)^{2}$. It grows at most exponentially:

$$
\exists C_{0}>0, \quad|x(t)|+|\xi(t)| \lesssim e^{C_{0} t}, \quad \forall t \geqslant 0 .
$$

As far as the Hartree kernel is concerned, two cases will be considered, leading to two different interesting phenomena:

- Smooth kernel: $K \in W^{3, \infty}\left(\mathbf{R}^{d}\right)$, with $K$ smooth near the origin.

- Homogeneous kernel: $K(x)=\lambda|x|^{-\gamma}$, with $\lambda \in \mathbf{R}$ and $0<\gamma<\min (2, d)$.

The second case includes the three-dimensional Schrödinger-Poisson, typically.

Remark 1.4. For several results (linearizable case - see definition below - or finite time propagation), the second assumption could be relaxed to $0<\gamma<\min (4, d)$ (energy-subcritical case). To simplify the presentation, we shall not discuss this extension.

We will focus on the first case in Section 2, in which we mostly revisit the results from [1]. In the rest of this introduction, we consider the homogeneous case. We seek the solution with the form

$$
\psi^{\varepsilon}(t, x)=\varepsilon^{-d / 4} u^{\varepsilon}\left(t, \frac{x-x(t)}{\sqrt{\varepsilon}}\right) e^{i(S(t)+\xi(t) \cdot(x-x(t))) / \varepsilon} .
$$

Here $S(t)$ is the classical Lagrangian action along the Hamiltonian flow generated by (1.4), given by

$$
S(t)=\int_{0}^{t}\left(\frac{1}{2}|\xi(s)|^{2}-V(s, x(s))\right) d s
$$


In terms of $u^{\varepsilon}=u^{\varepsilon}(t, y)$, (1.3) is equivalent

$$
i \partial_{t} u^{\varepsilon}+\frac{1}{2} \Delta u^{\varepsilon}=V^{\varepsilon}(t, y) u^{\varepsilon}+\lambda \varepsilon^{\alpha-\alpha_{c}}\left(|y|^{-\gamma} *\left|u^{\varepsilon}\right|^{2}\right) u^{\varepsilon}
$$

with the initial date $u^{\varepsilon}(0, y)=a(y)$, where

$$
\alpha_{c}=1+\frac{\gamma}{2}
$$

is a critical exponent and the time-dependent potential $V^{\varepsilon}(t, y)$ is given by

$$
V^{\varepsilon}(t, y)=\frac{1}{\varepsilon}(V(t, x(t)+\sqrt{\varepsilon} y)-V(t, x(t))-\sqrt{\varepsilon}\langle\nabla V(t, x(t)), y\rangle) .
$$

It reveals the first terms of the Taylor expansion of $V$ about the point $x(t)$. Passing formally to the limit, $V^{\varepsilon}$ converges to the Hessian of $V$ at $x(t)$ evaluated at $(y, y)$. Throughout the paper, we denote

$$
Q(t)=\operatorname{Hess} V(t, x(t))
$$

1.1. The linear case $\lambda=0$. Introduce the function

$$
\varphi_{\operatorname{lin}}^{\varepsilon}(t, x)=\varepsilon^{-d / 4} u_{\operatorname{lin}}\left(t, \frac{x-x(t)}{\sqrt{\varepsilon}}\right) e^{i(S(t)+\xi(t) \cdot(x-x(t))) / \varepsilon},
$$

where $u_{\text {lin }}$ solves

$$
i \partial_{t} u_{\operatorname{lin}}+\frac{1}{2} \Delta u_{\operatorname{lin}}=\frac{1}{2}\langle y, Q(t) y\rangle u_{\operatorname{lin}} \quad ; \quad u_{\operatorname{lin}}(0, y)=a(y) .
$$

Then the following lemma is well-known, see e.g. [2, 6, 8, 9, 10, 15, 16, 17] and references therein.

Lemma 1.5. Let $a \in \mathcal{S}\left(\mathbf{R}^{d}\right)$, and $\psi^{\varepsilon}$ solve (1.3) with $K=0$. There exist positive constants $C$ and $C_{1}$ independent of $\varepsilon$ such that

$$
\left\|\psi^{\varepsilon}(t)-\varphi_{\operatorname{lin}}^{\varepsilon}(t)\right\|_{L^{2}\left(\mathbf{R}^{d}\right)} \leqslant C \sqrt{\varepsilon} e^{C_{1} t} .
$$

In particular, there exists $c>0$ independent of $\varepsilon$ such that

$$
\sup _{0 \leqslant t \leqslant c \ln \frac{1}{\varepsilon}}\left\|\psi^{\varepsilon}(t)-\varphi_{\operatorname{lin}}^{\varepsilon}(t)\right\|_{L^{2}\left(\mathbf{R}^{d}\right) \underset{\varepsilon \rightarrow 0}{\longrightarrow} 0}^{\longrightarrow}
$$

1.2. The nonlinear case $\lambda \neq 0$. As in [6, we introduce two linear operators, which are essentially $\nabla$ and $x$, up to the wave packet scaling, in the moving frame:

$$
A^{\varepsilon}(t)=\sqrt{\varepsilon} \nabla-i \frac{\xi(t)}{\sqrt{\varepsilon}} \quad ; \quad B^{\varepsilon}(t)=\frac{x-x(t)}{\sqrt{\varepsilon}} .
$$

For $f \in \Sigma:=\left\{f \in H^{1}\left(\mathbf{R}^{d}\right) ; x f \in L^{2}\left(\mathbf{R}^{d}\right)\right\}$, we define

$$
\|f\|_{\mathcal{H}}=\|f\|_{L^{2}\left(\mathbf{R}^{d}\right)}+\left\|A^{\varepsilon} f\right\|_{L^{2}\left(\mathbf{R}^{d}\right)}+\left\|B^{\varepsilon} f\right\|_{L^{2}\left(\mathbf{R}^{d}\right)} .
$$


1.2.1. The subcritical case $\alpha>\alpha_{c}$. In this case, the solution of (1.3) is linearizable in the sense of [13]: $\varphi_{\text {lin }}$ yields a good approximation to $\psi^{\varepsilon}$, up to Ehrenfest time.

Proposition 1.6. Let $\lambda \in \mathbf{R}, 0<\gamma<\min (2, d)$ and $\alpha>\alpha_{c}$. Suppose that $a \in \mathcal{S}\left(\mathbf{R}^{d}\right)$ and $V$ satisfies Assumption 1.1. Then there exist positive constants $C, C_{1}, C_{2}$ independent of $\varepsilon$, and $\varepsilon_{0}>0$ such that for any $\left.\left.\varepsilon \in\right] 0, \varepsilon_{0}\right]$,

$$
\left\|\psi^{\varepsilon}(t)-\varphi_{\operatorname{lin}}^{\varepsilon}(t)\right\|_{\mathcal{H}} \leqslant C \varepsilon^{\kappa} e^{C_{1} t}, \quad 0 \leqslant t \leqslant C_{2} \ln \frac{1}{\varepsilon}, \quad \kappa=\min \left(\frac{1}{2}, \alpha-\alpha_{c}\right) .
$$

In particular, there exists a positive constant $c$ independent of $\varepsilon$ such that

$$
\sup _{0 \leqslant t \leqslant c \ln \frac{1}{\varepsilon}}\left\|\psi^{\varepsilon}(t)-\varphi_{\operatorname{lin}}^{\varepsilon}(t)\right\|_{\mathcal{H}} \underset{\varepsilon \rightarrow 0}{\longrightarrow} 0 .
$$

1.2.2. The critical case $\alpha=\alpha_{c}$. By passing formally to the limit $\varepsilon \rightarrow 0$, (1.7) can be written as

$$
i \partial_{t} u+\frac{1}{2} \Delta u=\frac{1}{2}\langle y, Q(t) y\rangle u+\lambda\left(|y|^{-\gamma} *|u|^{2}\right) u \quad ; \quad u(0, y)=a(y) .
$$

The Cauchy problem for (1.10) is addressed in $\$ 3.2$. For $\alpha=\alpha_{c}$, the solution to (1.3) is not linearizable: the nonlinearity affects the dynamics at leading order. For $k \in \mathbf{N}$, define $\left(\Sigma^{1}=\Sigma\right)$

$$
\Sigma^{k}=\left\{f \in L^{2}\left(\mathbf{R}^{d}\right) ;\|f\|_{\Sigma^{k}}:=\sum_{|\alpha|+|\beta| \leqslant k}\left\|x^{\alpha} \partial_{x}^{\beta} f\right\|_{L^{2}\left(\mathbf{R}^{d}\right)}<\infty\right\} .
$$

We prove:

Theorem 1.7. Let $\lambda \in \mathbf{R}, 0<\gamma<\min (2, d), \alpha=\alpha_{c}$ and $a \in \Sigma^{3}$. Suppose that $V$ satisfies Assumption 1.1. Let $u \in C\left(\mathbf{R}_{+} ; \Sigma^{3}\right)$ be the solution to (1.10) and

$$
\varphi^{\varepsilon}(t, x)=\varepsilon^{-d / 4} u\left(t, \frac{x-x(t)}{\sqrt{\varepsilon}}\right) e^{i(S(t)+\xi(t) \cdot(x-x(t))) / \varepsilon} .
$$

Then there exist positive constants $C, C_{1}, C_{2}$ independent of $\varepsilon$, and $\varepsilon_{0}>0$ such that for any $\left.\varepsilon \in] 0, \varepsilon_{0}\right]$,

$$
\left\|\psi^{\varepsilon}(t)-\varphi^{\varepsilon}(t)\right\|_{L^{2}\left(\mathbf{R}^{d}\right)} \leqslant C \sqrt{\varepsilon} \exp \left(C_{1} t\right), \quad 0 \leqslant t \leqslant C_{2} \ln \frac{1}{\varepsilon} .
$$

In particular, there exists a positive constant $c$ independent of $\varepsilon$ such that

$$
\sup _{0 \leqslant t \leqslant c \ln \frac{1}{\varepsilon}}\left\|\psi^{\varepsilon}(t)-\varphi^{\varepsilon}(t)\right\|_{L^{2}\left(\mathbf{R}^{d}\right)} \underset{\varepsilon \rightarrow 0}{\longrightarrow} 0 .
$$

Furthermore, if $a \in \Sigma^{4}$, then for the same constants $C_{1}, C_{2}$ as above,

$$
\left\|\psi^{\varepsilon}(t)-\varphi^{\varepsilon}(t)\right\|_{\mathcal{H}} \leqslant C_{3} \sqrt{\varepsilon} \exp \left(C_{1} t\right), \quad 0 \leqslant t \leqslant C_{2} \ln \frac{1}{\varepsilon} .
$$

Remark 1.8 (Notion of criticality). We will see in Section 2 that when $K$ is smooth near the origin, the critical value of $\alpha$ is $\alpha_{c}=1$, in sharp contrast with the homogeneous case. For more general Hartree kernels, the picture should be as follows. Assume that there exists $\lambda \in \mathbf{R} \backslash\{0\}, \gamma \geqslant 0, \delta>0$ such that

$$
K(x)=\lambda|x|^{-\gamma}+\mathcal{O}\left(|x|^{-\gamma+\delta}\right) \text { as } x \rightarrow 0,
$$

and that $K$ is smooth, bounded as well as its derivatives, away from the origin. Then we expect $\alpha_{c}=1+\gamma / 2$, with critical phenomena similar to the cases studied in this paper: like in the smooth kernel case if $\gamma=0$, and like in the homogeneous 
kernel case if $\gamma>0$ (since wave packets are extremely localized, the behavior of $K$ near the origin should be the only relevant one).

1.3. Nonlinear superposition. In this paragraph, we consider the critical case $\alpha=\alpha_{c}$. Suppose that initial data have the form

$$
\psi^{\varepsilon}(0, x)=\varepsilon^{-d / 4} a_{1}\left(\frac{x-x_{1}}{\sqrt{\varepsilon}}\right) e^{i\left(x-x_{1}\right) \cdot \xi_{1} / \varepsilon}+\varepsilon^{-d / 4} a_{2}\left(\frac{x-x_{2}}{\sqrt{\varepsilon}}\right) e^{i\left(x-x_{2}\right) \cdot \xi_{2} / \varepsilon},
$$

where $a_{1}, a_{2} \in \mathcal{S}\left(\mathbf{R}^{d}\right)$, and $\left(x_{1}, \xi_{1}\right) \neq\left(x_{2}, \xi_{2}\right)$. For $j \in\{1,2\},\left(x_{j}(t), \xi_{j}(t)\right)$ are the trajectories solutions to (1.4) with initial data $\left(x_{j}, \xi_{j}\right)$. Let $S_{j}(t)$ be the classical action associated with $\left(x_{j}(t), \xi_{j}(t)\right)$ given by (1.6) and $u_{j}$ be the solutions of (1.10) with initial data $a_{j}$. Assume the $\varphi_{j}^{\varepsilon}$ 's are defined as in (1.11), and $\psi^{\varepsilon} \in C\left(\mathbf{R}_{+} ; \Sigma\right)$ is the solution to (1.3). As in [6], for $f \in \Sigma$, define

$$
\|f\|_{\Sigma_{\varepsilon}}=\|f\|_{L^{2}\left(\mathbf{R}^{d}\right)}+\|\varepsilon \nabla f\|_{L^{2}\left(\mathbf{R}^{d}\right)}+\|x f\|_{L^{2}\left(\mathbf{R}^{d}\right)} .
$$

For bounded time, we have

Theorem 1.9. Let $0<\gamma<\min (2, d)$ and $a_{1}, a_{2} \in \mathcal{S}\left(\mathbf{R}^{d}\right)$. For any $T>0$ independent of $\varepsilon$,

$$
\sup _{0 \leqslant t \leqslant T}\left\|\psi^{\varepsilon}(t)-\varphi_{1}^{\varepsilon}(t)-\varphi_{2}^{\varepsilon}(t)\right\|_{\Sigma_{\varepsilon}}=\mathcal{O}\left(\varepsilon^{\frac{\gamma}{2(1+\gamma)}}\right) .
$$

When time becomes large, we can establish a superposition property for $d=1$, like in [6], where the condition $\gamma<\min (2, d)$ boils down to $\gamma<1$.

Theorem 1.10. Let $d=1,0<\gamma<1$ and $a_{1}, a_{2} \in \mathcal{S}\left(\mathbf{R}^{d}\right)$. Assume that $V$ does not depend on time, and define

$$
E_{j}=\frac{\xi_{j}^{2}}{2}+V\left(x_{j}\right)
$$

Suppose $E_{1} \neq E_{2}$. There exist positive constants $C, C_{1}, C_{2}$ independent of $\varepsilon$, and $\varepsilon_{0}>0$ such that for all $\left.\left.\varepsilon \in\right] 0, \varepsilon_{0}\right]$,

$$
\left\|\psi^{\varepsilon}(t)-\varphi_{1}^{\varepsilon}(t)-\varphi_{2}^{\varepsilon}(t)\right\|_{\Sigma_{\varepsilon}} \leqslant C \varepsilon^{\frac{\gamma}{2(1+\gamma)}} e^{C_{1} t}, \quad 0 \leqslant t \leqslant C_{2} \ln \frac{1}{\varepsilon} .
$$

In particular, there exists a positive constant $c$ independent of $\varepsilon$ such that

$$
\sup _{0 \leqslant t \leqslant c \ln \frac{1}{\varepsilon}}\left\|\psi^{\varepsilon}(t)-\varphi_{1}^{\varepsilon}(t)-\varphi_{2}^{\varepsilon}(t)\right\|_{\Sigma_{\varepsilon}} \underset{\varepsilon \rightarrow 0}{\longrightarrow} 0 .
$$

Notation. Throughout the paper, $C$ denotes a constant independent of $\varepsilon$ and $t$, whose value may change from one line to the other. For two positive numbers $a^{\varepsilon}$ and $b^{\varepsilon}$, the notation $a^{\varepsilon} \lesssim b^{\varepsilon}$ means that there exists $C>0$ independent of $\varepsilon$ such that for all $\varepsilon \in] 0,1], a^{\varepsilon} \leqslant C b^{\varepsilon}$.

\section{Smooth KeRNEL}

In this section, we shall assume that the kernel $K$ satisfies:

Assumption 2.1. The kernel is bounded as well as its first three derivatives, and smooth near the origin: for some neighborhood $\omega$ of the origin in $\mathbf{R}^{d}$,

$$
K \in W^{3, \infty}\left(\mathbf{R}^{d}\right) \cap C^{3}(\omega) .
$$

This assumption is similar to the one made in [1]. We first establish the local and global existence for the solution for (1.3) at the $L^{2}$ level. 
2.1. Construction of the exact solution. We prove that for fixed $\varepsilon>0$, (1.1) has a unique, global in time solution under Assumption 1.1. Since for such a result, $\varepsilon$ is irrelevant, we shall consider the case $\varepsilon=1$.

Lemma 2.2. Let $V$ satisfy Assumption 1.1, $K \in L^{\infty}\left(\mathbf{R}^{d}\right)$ and $\psi_{0} \in L^{2}\left(\mathbf{R}^{d}\right)$. There exists a unique solution $\psi \in C\left(\mathbf{R}_{+} ; L^{2}\left(\mathbf{R}^{d}\right)\right)$ to

$$
i \partial_{t} \psi+\frac{1}{2} \Delta \psi=V(t, x) \psi+\left(K *|\psi|^{2}\right) \psi \quad ; \quad \psi_{\mid t=0}=\psi_{0} .
$$

In addition, it satisfies $\|\psi(t)\|_{L^{2}\left(\mathbf{R}^{d}\right)}=\left\|\psi_{0}\right\|_{L^{2}\left(\mathbf{R}^{d}\right)}$ for all time $t \geqslant 0$.

Proof. Since $V$ may depend on time, we consider the more general Cauchy problem with a varying initial time:

$$
i \partial_{t} \psi+\frac{1}{2} \Delta \psi=V(t, x) \psi+\left(K *|\psi|^{2}\right) \psi \quad ; \quad \psi_{\mid t=s}=\psi_{s}
$$

with $\psi_{s} \in L^{2}\left(\mathbf{R}^{d}\right)$. In view of Assumption 1.1, the linear case generates a unitary semigroup ([11, 12]), which we denote by $U(t, s): \psi(t)=U(t, s) \psi_{s}$ when $K=0$.

In the nonlinear case, using Duhamel's formula, we can write (2.1) as

$$
\psi(t)=U(t, s) \psi_{s}-i \int_{s}^{t} U(t, \tau)\left(K *|\psi|^{2}\right) \psi(\tau) d \tau:=\Phi^{s}(\psi)(t) .
$$

For $s \geqslant 0$ and $T>0$, denote $I_{s, T}=[s, s+T]$ and introduce the space

$$
X_{s, T}=\left\{\psi \in C\left(I_{s, T}, L^{2}\left(\mathbf{R}^{d}\right)\right) ;\|\psi\|_{L^{\infty}\left(I_{s, T} ; L^{2}\left(\mathbf{R}^{d}\right)\right)} \leqslant 2\left\|\psi_{s}\right\|_{L^{2}\left(\mathbf{R}^{d}\right)}\right\} .
$$

Let $\psi, \psi_{1}, \psi_{2} \in X_{s, T}$. Using Hölder inequality, we have

$$
\begin{aligned}
\left\|\Phi^{S}(\psi)\right\|_{L^{\infty}\left(I_{s, T} ; L^{2}\left(\mathbf{R}^{d}\right)\right)} & \leqslant\left\|\psi_{s}\right\|_{L^{2}\left(\mathbf{R}^{d}\right)}+\left\|K *|\psi|^{2} \psi\right\|_{L^{1}\left(I_{s, T} ; L^{2}\left(\mathbf{R}^{d}\right)\right)} \\
& \leqslant\left\|\psi_{s}\right\|_{L^{2}\left(\mathbf{R}^{d}\right)}+T\|K\|_{L^{\infty}\left(\mathbf{R}^{d}\right)}\|\psi\|_{L^{\infty}\left(I_{T} ; L^{2}\left(\mathbf{R}^{d}\right)\right)}^{3} \\
& \leqslant\left\|\psi_{s}\right\|_{L^{2}\left(\mathbf{R}^{d}\right)}+8 T\|K\|_{L^{\infty}\left(\mathbf{R}^{d}\right)}\left\|\psi_{s}\right\|_{L^{2}\left(\mathbf{R}^{d}\right)}^{3} .
\end{aligned}
$$

Observe that

$$
\begin{aligned}
& K *\left|\psi_{1}\right|^{2} \psi_{1}-K *\left|\psi_{2}\right|^{2} \psi_{2} \\
= & \frac{1}{2} K *\left(\left|\psi_{1}\right|^{2}+\left|\psi_{2}\right|^{2}\right)\left(\psi_{1}-\psi_{2}\right)+\frac{1}{2} K *\left(\left|\psi_{1}\right|^{2}-\left|\psi_{2}\right|^{2}\right)\left(\psi_{1}+\psi_{2}\right) .
\end{aligned}
$$

Then by similar arguments as above, we have

$$
\left\|\Phi^{s}\left(\psi_{1}\right)-\Phi^{s}\left(\psi_{2}\right)\right\|_{L^{\infty}\left(I_{s, T} ; L^{2}\right)} \leqslant C T\|K\|_{L^{\infty}}\left\|\psi_{s}\right\|_{L^{2}}^{2}\left\|\psi_{1}-\psi_{2}\right\|_{L^{\infty}\left(I_{s, T} ; L^{2}\right)} .
$$

Taking $T$ small enough, we conclude $\Phi^{s}$ is a contraction from $X_{s, T}$ into itself, and there exists a unique local solution $\psi \in C\left(I_{s, T} ; L^{2}\left(\mathbf{R}^{d}\right)\right)$ to (2.1). By classical arguments, the $L^{2}$-norm of $\psi$ does not depend on time, and since $T$ depends only on $\left\|\psi_{s}\right\|_{L^{2}}$, the solution is global in time.

2.2. The general strategy. As in $[\underline{6}$, seek an approximate solution of the form

$$
\varphi^{\varepsilon}(t, x)=\varepsilon^{-d / 4} u\left(t, \frac{x-x(t)}{\sqrt{\varepsilon}}\right) e^{i(S(t)+\xi(t) \cdot(x-x(t))) / \varepsilon},
$$

for some profile $u$ independent of $\varepsilon$, and some function $S(t)$ to be determined. When $K=0, S$ is the classical action defined in (1.6). We will see that according to the value $\alpha$ in (1.3), the expression of $S$ may vary, accounting for nonlinear effects due to the presence of the Hartree nonlinearity. 
In the cases $\alpha=0,1 / 2,1$ and $\alpha>1$, we will see that we can write

$$
\begin{aligned}
i \varepsilon \partial_{t} \varphi^{\varepsilon}+\frac{\varepsilon^{2}}{2} \Delta \varphi^{\varepsilon} & -V \varphi^{\varepsilon}-\varepsilon^{\alpha}\left(K *\left|\varphi^{\varepsilon}\right|^{2}\right) \varphi^{\varepsilon}= \\
& \varepsilon^{-d / 4} e^{i(S(t)+\xi(t) \cdot(x-x(t))) / \varepsilon}\left(b_{0}+\sqrt{\varepsilon} b_{1}+\varepsilon b_{2}+\varepsilon r^{\varepsilon}\right),
\end{aligned}
$$

for $b_{0}, b_{1}, b_{2}$ independent of $\varepsilon$. The approximate solution $\varphi^{\varepsilon}$ will be determined by the conditions

$$
b_{0}=b_{1}=b_{2}=0 \text {. }
$$

The remaining factor $r^{\varepsilon}$ will account for the error between the exact solution $\psi^{\varepsilon}$ and the approximate solution $\varphi^{\varepsilon}$. Denote

$$
\phi(t, x)=S(t)+\xi(t) \cdot(x-x(t)) .
$$

The linear terms are computed as follows:

$$
\begin{aligned}
i \varepsilon \partial_{t} \varphi^{\varepsilon} & =\varepsilon^{-d / 4} e^{i \phi(t, x) / \varepsilon}\left(i \varepsilon \partial_{t} u-i \sqrt{\varepsilon} \dot{x}(t) \cdot \nabla u-u \partial_{t} \phi\right) . \\
\frac{\varepsilon^{2}}{2} \Delta \varphi^{\varepsilon} & =\varepsilon^{-d / 4} e^{i \phi(t, x) / \varepsilon}\left(\frac{\varepsilon}{2} \Delta u+i \sqrt{\varepsilon} \xi(t) \cdot \nabla u-\frac{|\xi(t)|^{2}}{2} u\right) .
\end{aligned}
$$

Here, as well as below, one should remember that the functions are assessed as in (2.2). Recalling that the relevant space variable for $u$ is

$$
y=\frac{x-x(t)}{\sqrt{\varepsilon}},
$$

we have:

$$
\partial_{t} \phi=\dot{S}(t)+\frac{d}{d t}(\xi(t) \cdot(x-x(t)))=\dot{S}(t)+\sqrt{\varepsilon} \dot{\xi}(t) \cdot y-\xi(t) \cdot \dot{x}(t) .
$$

For the linear potential term, we compute, in terms of the variable $y$,

$$
V \varphi^{\varepsilon}=V(t, x) \varepsilon^{-d / 4} e^{i \phi(t, x) / \varepsilon} u(t, y)=\varepsilon^{-d / 4} e^{i \phi(t, x) / \varepsilon} V(t, x(t)+y \sqrt{\varepsilon}) u(t, y),
$$

and we perform a Taylor expansion for $V$ about $(t, x(t))$ :

$$
\begin{aligned}
V(t, x(t)+y \sqrt{\varepsilon}) u(t, y)= & V(t, x(t)) u(t, y)+\sqrt{\varepsilon} y \cdot \nabla V(t, x(t)) u(t, y) \\
& +\frac{\varepsilon}{2}\left\langle y, \nabla^{2} V(t, x(t)) y\right\rangle u(t, y)+\varepsilon^{3 / 2} r_{V}^{\varepsilon}(t, y),
\end{aligned}
$$

with

$$
\left|r_{V}^{\varepsilon}(t, y)\right| \leqslant C\langle y\rangle^{3}|u(t, y)|
$$

for some $C$ independent of $\varepsilon, t$ and $y$, in view of Assumption 1.1. In the case $K=0$, we come up with the relations:

$$
\begin{aligned}
& b_{0}^{\operatorname{lin}}=-u\left(\dot{S}(t)-\xi(t) \cdot \dot{x}(t)+\frac{|\xi(t)|^{2}}{2}+V(t, x(t))\right) . \\
& b_{1}^{\operatorname{lin}}=-i(\dot{x}(t)-\xi(t)) \cdot \nabla u-y \cdot(\dot{\xi}(t)+\nabla V(t, x(t))) u . \\
& b_{2}^{\operatorname{lin}}=i \partial_{t} u+\frac{1}{2} \Delta u-\frac{1}{2}\left\langle y, \nabla^{2} V(t, x(t)) y\right\rangle u .
\end{aligned}
$$


For the nonlinear term, we compute similarly

$$
\begin{aligned}
\left(K *\left|\varphi^{\varepsilon}\right|^{2}\right)(t, x) & =\int K(x-z)\left|\varphi^{\varepsilon}(t, z)\right|^{2} d z \\
& =\varepsilon^{-d / 2} \int K(x-z)\left|u\left(t, \frac{z-x(t)}{\sqrt{\varepsilon}}\right)\right|^{2} d z \\
& =\int K(x-x(t)-z \sqrt{\varepsilon})|u(t, z)|^{2} d z .
\end{aligned}
$$

We have, in terms of the variable $y$ :

$$
\left(K *\left|\varphi^{\varepsilon}\right|^{2}\right)(t, x)=\int K((y-z) \sqrt{\varepsilon})|u(t, z)|^{2} d z .
$$

This is where the smoothness of $K$ near the origin becomes important: performing a Taylor expansion, we write

$$
\begin{aligned}
K((y-z) \sqrt{\varepsilon})= & K(0)+\sqrt{\varepsilon}(y-z) \cdot \nabla K(0)+\frac{\varepsilon}{2}\left\langle y-z, \nabla^{2} K(0)(y-z)\right\rangle \\
& +\varepsilon^{3 / 2} r_{K}^{\varepsilon}(y-z),
\end{aligned}
$$

with

$$
\left|r_{K}^{\varepsilon}(y-z)\right| \leqslant C\langle y-z\rangle^{3},
$$

for some $C$ independent of $\varepsilon, y$ and $z$. Therefore, using the conservation of mass,

$$
\begin{aligned}
\varepsilon^{d / 4} e^{-i \phi / \varepsilon} & \left(K *\left|\varphi^{\varepsilon}\right|^{2}\right) \varphi^{\varepsilon}(t, x)=K(0)\|a\|_{L^{2}}^{2} u(t, y)+\sqrt{\varepsilon}\|a\|_{L^{2}}^{2} y \cdot \nabla K(0) u(t, y) \\
& -\sqrt{\varepsilon} \nabla K(0) \cdot G(t) u(t, y)+\frac{\varepsilon}{2}\left\langle y, \nabla^{2} K(0) y\right\rangle\|a\|_{L^{2}}^{2} u(t, y) \\
& +\frac{\varepsilon}{2} \int\left\langle z, \nabla^{2} K(0) z\right\rangle|u(t, z)|^{2} d z \times u(t, y) \\
& -\varepsilon\left\langle\nabla^{2} K(0) G(t), y\right\rangle u(t, y),
\end{aligned}
$$

where the notation $G(t)$ stands for

$$
G(t)=\int_{\mathbf{R}^{d}} z|u(t, z)|^{2} d z
$$

We then discuss the outcome in (2.3) according to the value of $\alpha$, on a formal level. We present the strategy to justify the approximation in the case $\alpha=0$ only, since this case contains all the arguments needed to treat the other cases.

2.3. Subcritical case: $\alpha>1$. When $\alpha>1$, by have $b_{j}=b_{j}^{\text {lin }}$ for $j=0,1,2$, and

$$
r^{\varepsilon}=\sqrt{\varepsilon} r_{V}^{\varepsilon}+\varepsilon^{\alpha-1}\left(K *\left|\varphi^{\varepsilon}\right|^{2}\right) \varphi^{\varepsilon} .
$$

Solving the equations $b_{0}^{\text {lin }}=b_{1}^{\text {lin }}=b_{2}^{\text {lin }}$ leads to the approximate solution $\varphi_{\text {lin }}^{\varepsilon}$ defined in Section 1.1

2.4. The critical case: $\alpha=1$. When $\alpha=1$, we still have $b_{j}=b_{j}^{\text {lin }}$ for $j=0,1$, but the expression for $b_{2}$ is altered:

$$
b_{2}=i \partial_{t} u+\frac{1}{2} \Delta u-\frac{1}{2}\left\langle y, \nabla^{2} V(t, x(t)) y\right\rangle u-K(0)\|a\|_{L^{2}}^{2} u .
$$

The equation $b_{2}=0$ is the linear envelope equation (1.9), plus a constant potential, $K(0)\|a\|_{L^{2}}^{2}$. We infer

$$
u(t, y)=u_{\operatorname{lin}}(t, y) e^{-i t K(0)\|a\|_{L^{2}}^{2}} .
$$


The presence of this phase shift accounts for nonlinear effects at leading order in the approximate wave packet $\varphi^{\varepsilon}$. For the remainder term, we have:

$$
\begin{aligned}
r^{\varepsilon}(t, y) & =\sqrt{\varepsilon} r_{V}^{\varepsilon}(t, y)+\sqrt{\varepsilon}\|a\|_{L^{2}}^{2} y \cdot \nabla K(0) u(t, y) \\
& -\sqrt{\varepsilon} \nabla K(0) \cdot G(t) u(t, y)+\frac{\varepsilon}{2}\left\langle y, \nabla^{2} K(0) y\right\rangle\|a\|_{L^{2}}^{2} u(t, y) \\
& +\frac{\varepsilon}{2} \int\left\langle z, \nabla^{2} K(0) z\right\rangle|u(t, z)|^{2} d z \times u(t, y) \\
& -\varepsilon\left\langle\nabla^{2} K(0) G(t), y\right\rangle u(t, y),
\end{aligned}
$$

and we infer the (rough) pointwise estimate

$$
\left|r^{\varepsilon}(t, y)\right| \leqslant C \sqrt{\varepsilon}\langle y\rangle^{3}|u(t, y)|\left(1+\|u(t)\|_{\Sigma}^{2}\right) .
$$

2.5. A supercritical case: $\alpha=1 / 2$. For $\alpha<1$, we have to assume either $\alpha=1 / 2$ or $\alpha=0$ in order to derive functions $b_{j}$ which do not depend on $\varepsilon$. For the simplicity of the presentation, we therefore stick to these cases, but essentially, the case $1 / 2<\alpha<1$ is treated like the case $\alpha=1 / 2$, and the case $0<\alpha<1 / 2$ like the case $\alpha=0$.

In the case $\alpha=1 / 2$, we still have $b_{0}=b_{0}^{\text {lin }}$, but now with

$$
\begin{aligned}
& b_{1}=-i(\dot{x}(t)-\xi(t)) \cdot \nabla u-y \cdot(\dot{\xi}(t)+\nabla V(t, x(t))) u-K(0)\|a\|_{L^{2}}^{2} u . \\
& b_{2}=i \partial_{t} u+\frac{1}{2} \Delta u-\frac{1}{2}\left\langle y, \nabla^{2} V(t, x(t)) y\right\rangle u-\|a\|_{L^{2}}^{2} y \cdot \nabla K(0) u+\nabla K(0) \cdot G(t) u .
\end{aligned}
$$

At this stage, it is easy to convince oneself that the equations $b_{0}=b_{1}=b_{2}=0$ are not compatible in general (if one wants to consider a non-zero solution $u$ ). Therefore, we modify our strategy, in order to allow $b_{0}$ to depend on $\varepsilon$, so we can upgrade the last factor in $b_{1}$ to $b_{0}$. This leads to:

$$
\begin{aligned}
& b_{0}^{\varepsilon}=-u\left(\dot{S}(t)-\xi(t) \cdot \dot{x}(t)+\frac{|\xi(t)|^{2}}{2}+V(t, x(t))+\sqrt{\varepsilon} K(0)\|a\|_{L^{2}}^{2}\right) . \\
& b_{1}=-i(\dot{x}(t)-\xi(t)) \cdot \nabla u-y \cdot(\dot{\xi}(t)+\nabla V(t, x(t))) u . \\
& b_{2}=i \partial_{t} u+\frac{1}{2} \Delta u-\frac{1}{2}\left\langle y, \nabla^{2} V(t, x(t)) y\right\rangle u-\|a\|_{L^{2}}^{2} y \cdot \nabla K(0) u+\nabla K(0) \cdot G(t) u .
\end{aligned}
$$

Keeping $(x(t), \xi(t))$ solution to the Hamiltonian flow (1.4) leads to the $\varepsilon$-dependent action:

$$
S^{\varepsilon}(t)=\int_{0}^{t}\left(\frac{1}{2}|\xi(s)|^{2}-V(s, x(s))\right) d s-t \sqrt{\varepsilon} K(0)\|a\|_{L^{2}\left(\mathbf{R}^{d}\right)}^{2} .
$$

The equation $b_{1}=0$ is then fulfilled as soon as we consider the standard Hamiltonian flow. The equation $b_{2}=0$ is an envelope equation, which is nonlinear since $G$ is a nonlinear function of $u$. Note however that this yields a purely time-dependent potential. Up to the time-dependent gauge transform

$$
u(t, y) \mapsto u(t, y) \exp \left(i \int_{0}^{t} \nabla K(0) \cdot G(s) d s\right),
$$

which preserves the modulus of the unknown, hence $G$, the equation for $u$ becomes a linear profile equation. Finally, we still have a remainder term satisfying (2.4). 
2.6. Another supercritical case: $\alpha=0$. This case corresponds to the one studied in [1]. We consider a more general framework though, since for instance we do not assume that the kernel $K$ is radially symmetric. We now have

$$
\begin{aligned}
b_{0}= & -u\left(\dot{S}(t)-\xi(t) \cdot \dot{x}(t)+\frac{|\xi(t)|^{2}}{2}+V(t, x(t))+K(0)\|a\|_{L^{2}}^{2}\right), \\
b_{1}= & -i(\dot{x}(t)-\xi(t)) \cdot \nabla u-y \cdot(\dot{\xi}(t)+\nabla V(t, x(t))) u-\|a\|_{L^{2}}^{2} y \cdot \nabla K(0) u \\
& +\nabla K(0) \cdot G(t) u, \\
b_{2}= & i \partial_{t} u+\frac{1}{2} \Delta u-\frac{1}{2}\langle y, M(t) y\rangle u+\left\langle\nabla^{2} K(0) G(t), y\right\rangle u \\
& -\frac{1}{2} \int\left\langle z, \nabla^{2} K(0) z\right\rangle|u(t, z)|^{2} d z \times u,
\end{aligned}
$$

where we have denoted

$$
M(t)=\|a\|_{L^{2}\left(\mathbf{R}^{d}\right)}^{2} \nabla^{2} K(0)+\nabla_{x}^{2} V(t, x(t)) .
$$

Note that $M \in L_{t}^{\infty}\left(\mathbf{R}_{+}\right)$. We will assume

$$
\nabla K(0)=0 \text {, }
$$

so $b_{1}=0$ as soon as $(x(t), \xi(t))$ satisfies (1.4). This assumption is a consequence of the framework in [1], since the authors suppose $K(x)=F(|x|)$ with $F$ even. Note that the slightly more general assumption $K(x)=K(-x)$ is physically relevant, in the sense that in that case, an energy can be associated to the Hartree nonlinearity (see e.g. [7]):

$$
\iint K(x-y)\left|\psi^{\varepsilon}(t, x)\right|^{2}\left|\psi^{\varepsilon}(t, y)\right|^{2} d x d y
$$

In the case where $K$ is even, we obviously have $\nabla K(0)=0$. We then consider the Hamiltonian flow (1.4), the modified action

$$
S(t)=\int_{0}^{t}\left(\frac{1}{2}|\xi(s)|^{2}-V(s, x(s))\right) d s-t K(0)\|a\|_{L^{2}\left(\mathbf{R}^{d}\right)}^{2},
$$

and the envelope equation $b_{2}=0$. The remainder term still satisfies (2.4).

Remark 2.3. Note that the Wigner measure of $\psi^{\varepsilon}$ is not affected by the nonlinearity:

$$
w(t, x, \xi)=\|a\|_{L^{2}\left(\mathbf{R}^{d}\right)}^{2} \delta(x-x(t)) \otimes \delta(\xi-\xi(t)),
$$

in the four cases $\alpha>1, \alpha=1, \alpha=1 / 2$ and $\alpha=0$, even though we have seen that the Hartree nonlinearity does affect the leading order behavior of the wave function.

2.7. Sketch of the proof in the case $\alpha=0$. We want to construct a solution to

$$
\begin{aligned}
i \partial_{t} u+\frac{1}{2} \Delta u= & \frac{1}{2}\langle y, M(t) y\rangle u-\left\langle\nabla^{2} K(0) G(t), y\right\rangle u \\
& +\frac{1}{2} \int\left\langle z, \nabla^{2} K(0) z\right\rangle|u(t, z)|^{2} d z \times u,
\end{aligned}
$$

with initial datum $a$. Introduce the solution to

$$
i \partial_{t} v+\frac{1}{2} \Delta v=\frac{1}{2}\langle y, M(t) y\rangle v-\left\langle\nabla^{2} K(0) G(t), y\right\rangle v \quad ; \quad v(0, y)=a(y) .
$$


The functions $u$ and $v$ solve the same equation, up to one term which can be absorbed by the gauge transform $u(t, y)=v(t, y) \exp (i \theta(t))$, where

$$
\theta(t)=-\frac{1}{2} \int_{0}^{t} \int_{\mathbf{R}^{d}}\left\langle z, \nabla^{2} K(0) z\right\rangle|u(s, z)|^{2} d z d s .
$$

Since this gauge transform does not affect the modulus of the solution, one should consider that in (2.7),

$$
G(t)=\int_{\mathbf{R}^{d}} z|v(t, z)|^{2} d z .
$$

Lemma 2.4. Let $k \geqslant 1$ and assume that $a \in \Sigma^{k}$. Then (2.7) has a unique solution $v \in C\left(\mathbf{R}_{+} ; \Sigma^{k}\right)$, and there exists $C$ such that

$$
\|v(t)\|_{\Sigma^{k}} \leqslant C e^{C t}, \quad t \geqslant 0 .
$$

As a consequence, (2.6) has a unique solution, which possesses the same properties.

Remark 2.5. The $\Sigma$ regularity is the least one has to demand in this result, for the gauge $\theta$ to be well defined (and the harmonic oscillator rotates the phase space, so the regularity must be the same in space and frequency).

Sketch of the proof. The main difficulty is that since the last term in the equation involves a time dependent potential which is unbounded in $y$, it cannot be treated as a perturbation. So to construct a local solution, we modify the standard Picard iterative scheme, to consider

$$
i \partial_{t} v_{n}+\frac{1}{2} \Delta v_{n}=\frac{1}{2}\langle y, M(t) y\rangle v_{n}+\left\langle\nabla^{2} K(0) G_{n-1}(t), y\right\rangle v_{n}, n \geqslant 1,
$$

with $v_{n \mid t=0}=a$ for all $n, v_{0}(t, y)=a(y)$, and

$$
G_{k}(t)=\int_{\mathbf{R}^{d}} z\left|v_{k}(s, z)\right|^{2} d s .
$$

At each step, we solve a linear equation, with a time dependent potential which is at most quadratic: if $G_{n-1} \in L_{\mathrm{loc}}^{\infty}\left(\mathbf{R}_{+}\right)$, 12 ensures the existence of $v_{n} \in$ $C\left(\mathbf{R}_{+} ; L^{2}\left(\mathbf{R}^{d}\right)\right)$. Applying the operators $y$ and $\nabla_{y}$ to $(2.8)$ shows that $v_{n} \in$ $C\left(\mathbf{R}_{+} ; \Sigma\right)$, hence $G_{n} \in L_{\text {loc }}^{\infty}\left(\mathbf{R}_{+}\right)$. To prove the convergence of this scheme we need more precise (uniform in $n$ ) estimates. Direct computations show that

$$
\dot{G}_{n}(t)=\operatorname{Im} \int_{\mathbf{R}^{d}} \overline{v_{n}}(t, z) \nabla v_{n}(t, z) d z,
$$

and

$$
\ddot{G}_{n}(t)+M(t) G_{n}(t)=\nabla^{2} K(0)\|a\|_{L^{2}\left(\mathbf{R}^{d}\right)}^{2} G_{n-1}, \quad n \geqslant 1 .
$$

Let $f_{n}(t)=\left|\dot{G}_{n}(t)\right|^{2}+\left|G_{n}(t)\right|^{2}$. We have

$$
\dot{f}_{n}(t) \leqslant 2\left|\dot{G}_{n}(t)\right|\left|\ddot{G}_{n}(t)\right|+2\left|\dot{G}_{n}(t)\right|\left|G_{n}(t)\right| \leqslant C f_{n}(t)+C\left|G_{n-1}(t)\right|^{2},
$$

for some $C$ independent of $t$ and $n$. We infer that there exists $C_{0}$ independent of $t \geqslant 0$ and $n$ such that

$$
f_{n}(t)=\left|\dot{G}_{n}(t)\right|^{2}+\left|G_{n}(t)\right|^{2} \leqslant C_{0} e^{C_{0} t} .
$$

By using energy estimates (applying the operators $y$ and $\nabla_{y}$ successively to the equation), we infer that there exists $C_{1}$ independent of $t \geqslant 0$ and $n$ such that

$$
\left\|v_{n}(t)\right\|_{\Sigma^{k}} \leqslant C_{1} e^{C_{1} t} .
$$


The convergence of the sequence $v_{n}$ then follows: by a standard fixed point argument, $v_{n}$ converges in $C([0, T] ; \Sigma)$ if $T>0$ is sufficiently small. By using energy estimates, and (exponential) a priori bounds for $G$, we infer the exponential control stated in the lemma, and hence global existence.

Remark 2.6. The above computations show that for $v$, the function $G$ satisfies

$$
\begin{aligned}
& \ddot{G}(t)+\nabla_{x}^{2} V(t, x(t)) G(t)=0 \\
& G(0)=\int_{\mathbf{R}^{d}} z|a(z)|^{2} d z \quad ; \quad \dot{G}(0)=\operatorname{Im} \int_{\mathbf{R}^{d}} \bar{a}(z) \nabla a(z) d z .
\end{aligned}
$$

In [1, the authors proved that if the initial data $a \in \Sigma^{3}$ is such that

$$
\int_{\mathbf{R}^{d}} z|a(z)|^{2} d z=\operatorname{Im} \int_{\mathbf{R}^{d}} \bar{a}(z) \nabla a(z) d z=0,
$$

then $\int z|u(t, z)|^{2} d z=0$ for all time. The above ODE gives a simple explanation of that property. Note that up to changing $a$ to $b$ with

$$
b(y)=a\left(y-y_{0}\right) e^{i y \cdot \eta_{0}}
$$

for $y_{0}$ and $\eta_{0}$ which can be computed explicitly, that is up to a translation in the phase space, these two assumptions are satisfied. However, the external potential is modified, and it is not so easy to keep track of the geometric meaning of the approximation. This is why we have chosen to sketch a direct approach here, which also shows that (2.7) is more nonlinear than it may seem. Note finally that because of the term $G$, working in $L^{2}$ only would not be possible.

To conclude, we have:

Proposition 2.7. Let $a \in \Sigma^{3}, \alpha=0$. Suppose $V$ satisfies Assumption 1.1, and $K$ satisfies Assumption 2.1 and $\nabla K(0)=0$. Assume $\varphi^{\varepsilon}$ is given by (2.2), where the action is given by (2.5) and the envelope is given by (2.6). Then there exists a positive constant $C$ independent of $\varepsilon$ such that

$$
\left\|\psi^{\varepsilon}(t)-\varphi^{\varepsilon}(t)\right\|_{L^{2}\left(\mathbf{R}^{d}\right)} \leqslant C \sqrt{\varepsilon} e^{e^{C t}}, \quad t \geqslant 0 .
$$

In particular, there exists $c>0$ independent of $\varepsilon$ such that

$$
\sup _{0 \leqslant t \leqslant c \ln \ln \frac{1}{\varepsilon}}\left\|\psi^{\varepsilon}(t)-\varphi^{\varepsilon}(t)\right\|_{L^{2}\left(\mathbf{R}^{d}\right)} \underset{\varepsilon \rightarrow 0}{\longrightarrow} 0 .
$$

Proof. First, we change the unknown function $\psi^{\varepsilon}$ to $u^{\varepsilon}$ through the bijective change of unknown function

$$
\psi^{\varepsilon}(t, x)=\varepsilon^{-d / 4} u^{\varepsilon}\left(t, \frac{x-x(t)}{\sqrt{\varepsilon}}\right) e^{i(S(t)+\xi(t) \cdot(x-x(t))) / \varepsilon},
$$

where $S$ is given by (2.5). Then (1.3) (with $\alpha=0$ ) is equivalent to:

$$
i \partial_{t} u^{\varepsilon}+\frac{1}{2} \Delta u^{\varepsilon}=V^{\varepsilon} u^{\varepsilon}+\left(K^{\varepsilon} *\left|u^{\varepsilon}\right|^{2}\right) u^{\varepsilon} \quad ; \quad u^{\varepsilon}(0, y)=a(y),
$$

where

$$
\begin{aligned}
V^{\varepsilon}(t, y) & =\frac{1}{\varepsilon}(V(t, x(t)+y \sqrt{\varepsilon})-V(t, x(t))-\sqrt{\varepsilon} y \cdot \nabla V(t, x(t))), \\
K^{\varepsilon}(y) & =\frac{1}{\varepsilon}(K(y \sqrt{\varepsilon})-K(0)) .
\end{aligned}
$$


We have, in view of Assumption 1.1 Assumption 2.1. the property $\nabla K(0)=0$, and Taylor's formula,

$$
\left|V^{\varepsilon}(t, y)\right|+\left|K^{\varepsilon}(y)\right| \leqslant C|y|^{2},
$$

for some constant $C$ independent of $\varepsilon, t$ and $y$. We already know from Lemma 2.2 that $u^{\varepsilon} \in C\left(\mathbf{R}_{+} ; L^{2}\right)$, with $\left\|u^{\varepsilon}(t)\right\|_{L^{2}}=\|a\|_{L^{2}}$. Proceeding in the same way as in the proof of Lemma 2.4, we can also prove that $u^{\varepsilon} \in C\left(\mathbf{R}_{+} ; \Sigma^{3}\right)$ and that there exists $C$ such that

$$
\left\|u^{\varepsilon}(t)\right\|_{\Sigma^{3}} \leqslant C e^{C t} .
$$

Set $w^{\varepsilon}=u^{\varepsilon}-u$ : we have $\left\|w^{\varepsilon}(t)\right\|_{L^{2}}=\left\|\psi^{\varepsilon}(t)-\varphi^{\varepsilon}(t)\right\|_{L^{2}}$, and $w^{\varepsilon}$ solves

$$
i \partial_{t} w^{\varepsilon}+\frac{1}{2} \Delta w^{\varepsilon}=V^{\varepsilon} w^{\varepsilon}+\left(K^{\varepsilon} *\left|u^{\varepsilon}\right|^{2}\right) u^{\varepsilon}-\left(K^{\varepsilon} *|u|^{2}\right) u-r^{\varepsilon} \quad ; \quad w_{\mid t=0}^{\varepsilon}=0,
$$

where $r^{\varepsilon}$ satisfies the pointwise estimate (2.4). Write

$$
\left(K^{\varepsilon} *\left|u^{\varepsilon}\right|^{2}\right) u^{\varepsilon}-\left(K^{\varepsilon} *|u|^{2}\right) u=\left(K^{\varepsilon} *\left|u^{\varepsilon}\right|^{2}\right) w^{\varepsilon}+\left(K^{\varepsilon} *\left(\left|u^{\varepsilon}\right|^{2}-|u|^{2}\right)\right) u,
$$

so the standard $L^{2}$ estimate yields

$$
\left\|w^{\varepsilon}(t)\right\|_{L^{2}} \leqslant \int_{0}^{t}\left\|\left(K^{\varepsilon} *\left(\left|u^{\varepsilon}\right|^{2}-|u|^{2}\right)\right) u(s)\right\|_{L^{2}} d s+\int_{0}^{t}\left\|r^{\varepsilon}(s)\right\|_{L^{2}} d s .
$$

Since $\left|u^{\varepsilon}\right|^{2}-|u|^{2}=2 \operatorname{Re}\left(\bar{u} w^{\varepsilon}\right)+\left|w^{\varepsilon}\right|^{2}$, and $\left\|u^{\varepsilon}(t)\right\|_{\Sigma^{2}}+\|u(t)\|_{\Sigma^{3}} \leqslant C e^{C t}$, we come up with

$$
\left\|w^{\varepsilon}(t)\right\|_{L^{2}} \leqslant C \int_{0}^{t} e^{C s}\left\|w^{\varepsilon}(s)\right\|_{L^{2}} d s+C \sqrt{\varepsilon} \int_{0}^{t} e^{C s} d s .
$$

Gronwall lemma yields

$$
\left\|w^{\varepsilon}(t)\right\|_{L^{2}} \leqslant C \sqrt{\varepsilon} e^{e^{C t}}
$$

and the result follows.

Remark 2.8. It is quite surprising that even in a supercritical case, the approximation can be proven so simply, eventually by a Gronwall type argument. This is in sharp contrast with supercritical WKB analysis for the nonlinear Schrödinger equation (see [4]). On the other hand, we had to use the a priori control of the approximate solution $u$ and of the exact solution $u^{\varepsilon}$ : in subcritical or critical cases, controlling the approximate solution is sufficient in general, as illustrated below in the case of homogeneous kernels.

Remark 2.9. In the cases $\alpha=1 / 2, \alpha=1$, and $\alpha>1$, a similar statement can be proved, and the time of validity is improved, in the sense that the $c \ln \ln 1 / \varepsilon$ in the end of Proposition 2.7 can be replaced by $c \ln 1 / \varepsilon$. More precisely, the error estimate will be:

- $\alpha>1:\left\|\psi^{\varepsilon}(t)-\varphi_{\text {lin }}^{\varepsilon}(t)\right\|_{L^{2}} \leqslant C \varepsilon^{\kappa} e^{C t}$ with $\kappa=\min (1 / 2, \alpha-1)$.

- $\alpha=1:\left\|\psi^{\varepsilon}(t)-\varphi^{\varepsilon}(t)\right\|_{L^{2}} \leqslant C \sqrt{\varepsilon} e^{C t}$, with $\varphi^{\varepsilon}$ as in $\$ 2.4$.

- $\alpha=\frac{1}{2}:\left\|\psi^{\varepsilon}(t)-\varphi^{\varepsilon}(t)\right\|_{L^{2}} \leqslant C \sqrt{\varepsilon} e^{C t} \exp \left(\sqrt{\varepsilon} e^{C t}\right)$, with $\varphi^{\varepsilon}$ as in $\$ 2.5$, 


\section{HOMOGENEOUS KERNEL: TECHNICAL BACKGROUND}

In this section, we present some general technical tools which will be used in the proofs of the main results in the case of an homogeneous kernel. In particular, we establish the global well-posedness for (1.10), and estimate the evolution of weighted Sobolev norms of the solution over large time:

Proposition 3.1. Let $\lambda \in \mathbf{R}$ and $0<\gamma<\min (2, d)$. Suppose $V$ satisfies Assumption 1.1 and the initial data $a \in L^{2}\left(\mathbf{R}^{d}\right)$. Then there exists a unique solution $u \in C\left(\mathbf{R}_{+} ; L^{2}\left(\mathbf{R}^{d}\right)\right) \cap L_{\mathrm{loc}}^{8 / \gamma}\left(\mathbf{R}_{+}, L^{4 d /(2 d-\gamma)}\left(\mathbf{R}^{d}\right)\right)$ to (1.10). If in addition $a \in \Sigma^{k}$ for some $k \in \mathbf{N}$, then $u \in C\left(\mathbf{R}_{+} ; \Sigma^{k}\right)$, and there exists $C=C(k)$ such that

$$
\|u(t)\|_{\Sigma^{k}} \leqslant C e^{C t}, \quad \forall t \geqslant 0
$$

3.1. Strichartz estimates. Before studying the semi-classical limit, we recall some known facts and establish technical results.

Definition 3.2. A pair $(q, r)$ is admissible if $2 \leqslant r<\frac{2 d}{d-2}(2 \leqslant q \leqslant \infty$ if $d=1$, $2 \leqslant q<\infty$ if $d=2$ ) and

$$
\frac{2}{q}=d\left(\frac{1}{2}-\frac{1}{r}\right):=\delta(r)
$$

Following [14, 19, 25], Strichartz estimates are available for the Schrödinger equation without external potential. Thanks to the construction of the parametrix performed in [11, 12, similar results are available in the presence of an external potential satisfying

Assumption 3.3. $W \in L_{\mathrm{loc}}^{\infty}\left(\mathbf{R}_{+} \times \mathbf{R}^{d}\right)$ is a smooth with respect to $x$ for all $t \geqslant 0$ : $x \mapsto W(t, x)$ is a $C^{\infty}$ map. Moreover, it is subquadratic in $x$ :

$$
\forall \beta \in \mathbf{N}^{d},|\beta| \geqslant 2, \partial_{x}^{\beta} W \in L^{\infty}\left(\mathbf{R}_{+} \times \mathbf{R}^{d}\right) .
$$

Define $U^{\varepsilon}(t, s)$ the semigroup as $u^{\varepsilon}(t, x)=U^{\varepsilon}(t, s) \phi(x)$, where

$$
i \varepsilon \partial_{t} u^{\varepsilon}+\frac{\varepsilon^{2}}{2} \Delta u^{\varepsilon}=W(t, x) u^{\varepsilon} ; \quad u^{\varepsilon}(s, x)=\phi(x) .
$$

From [11, 12, it has the following properties:

- $U^{\varepsilon}(t, t)=\mathrm{Id}$.

- The map $(t, s) \mapsto U^{\varepsilon}(t, s)$ is strongly continuous.

- $U^{\varepsilon}(t, \tau) U^{\varepsilon}(\tau, s)=U^{\varepsilon}(t, s)$.

- $U^{\varepsilon}(t, s)^{*}=U^{\varepsilon}(t, s)^{-1}$.

- $U^{\varepsilon}(t, s)$ is unitary on $L^{2}$ norm: $\left\|U^{\varepsilon}(t, s) \phi\right\|_{L^{2}\left(\mathbf{R}^{d}\right)}=\|\phi\|_{L^{2}\left(\mathbf{R}^{d}\right)}$.

- There exist $\delta, C>0$ independent of $\varepsilon \in] 0,1]$ such that for all $t, s \geqslant 0$ with $|t-s|<\delta$

$$
\left\|U^{\varepsilon}(t, 0) U^{\varepsilon}(s, 0)^{*} \phi\right\|_{L^{\infty}\left(\mathbf{R}^{d}\right)} \leqslant \frac{C}{(\varepsilon|t-s|)^{d / 2}}\|\phi\|_{L^{1}\left(\mathbf{R}^{d}\right)} .
$$

Scaled Strichartz estimates follow from the above dispersive relation:

Proposition 3.4 (Scaled Strichartz estimates). Let $U^{\varepsilon}(t, s)$ defined as above. There exists $\delta>0$ independent of $\varepsilon$ such that the following holds.

(1) For any admissible pair $(q, r)$, there exists $C(q)$ independent of $\varepsilon$ such that

$$
\varepsilon^{1 / q}\left\|U^{\varepsilon}(\cdot, s) \phi\right\|_{L^{q}\left([s, s+\delta] ; L^{r}\left(\mathbf{R}^{d}\right)\right)} \leqslant C(q)\|\phi\|_{L^{2}\left(\mathbf{R}^{d}\right)}, \quad \forall \phi \in L^{2}\left(\mathbf{R}^{d}\right), \quad \forall s \geqslant 0 .
$$


(2) For $s \in \mathbf{R}$, denote

$$
D_{s}^{\varepsilon}(F)(t, x)=\int_{s}^{t} U^{\varepsilon}(t, s) F(s, x) d s,
$$

and $I=[s, s+\eta]$. For all admissible pairs $\left(q_{1}, r_{1}\right)$ and $\left(q_{2}, r_{2}\right)$, there exists $C\left(q_{1}, q_{2}\right)$ independent of $\varepsilon$ and $s \geqslant 0$ such that

$$
\varepsilon^{1 / q_{1}+1 / q_{2}}\left\|D_{s}^{\varepsilon}(F)\right\|_{L^{q_{1}}\left(I ; L^{r_{1}}\left(\mathbf{R}^{d}\right)\right)} \leqslant C\left(q_{1}, q_{2}\right)\|F\|_{L^{q_{2}^{\prime}\left(I ; L^{r_{2}^{\prime}}\left(\mathbf{R}^{d}\right)\right)}}
$$

for all $F \in L^{q_{2}^{\prime}}\left(I ; L^{r_{2}^{\prime}}\left(\mathbf{R}^{d}\right)\right)$ and $0 \leqslant \eta \leqslant \delta$. Here $\frac{1}{q_{2}}+\frac{1}{q_{2}^{\prime}}=1$ and $\frac{1}{r_{2}}+\frac{1}{r_{2}^{\prime}}=1$.

This statement will be used in the two cases $\varepsilon=1$ (for the envelope equation (1.10) ), and $\varepsilon \in] 0,1]$ (to justify the approximation of the exact solution $\psi^{\varepsilon}$ ).

3.2. Global existence in $L^{2}$. We consider a rather general potential $W$ satisfying Assumption 3.3, and consider the Cauchy problem on $\mathbf{R}_{+} \times \mathbf{R}^{d}$ :

$$
i \partial_{t} v+\frac{1}{2} \Delta v=W(t, y) v+\lambda|y|^{-\gamma} *|v|^{2} v \quad ;\left.\quad v\right|_{t=s}=v_{s} .
$$

This form includes the cases of the exact solution $\psi^{\varepsilon}$ in (1.3) as well as the envelope equation (1.10). We establish global existence for (3.1) in the $L^{2}$-subcritical case $0<\gamma<\min (2, d)$, yielding the first part of Proposition 3.1.

Lemma 3.5. Let $\lambda \in \mathbf{R}, 0<\gamma<\min (2, d)$. Suppose $W$ satisfies Assumption 3.3. Then for $s=0$ and $v_{0} \in L^{2}\left(\mathbf{R}^{d}\right)$, 3.1) has a unique solution

$$
v \in C\left(\mathbf{R}_{+} ; L^{2}\left(\mathbf{R}^{d}\right)\right) \cap L_{\text {loc }}^{8 / \gamma}\left(\mathbf{R}_{+}, L^{4 d /(2 d-\gamma)}\left(\mathbf{R}^{d}\right)\right) .
$$

In addition, the $L^{2}$ norm of $v$ is conserved:

$$
\|v(t)\|_{L^{2}\left(\mathbf{R}^{d}\right)}=\left\|v_{0}\right\|_{L^{2}\left(\mathbf{R}^{d}\right)}, \forall t \geqslant 0 .
$$

Proof. By Duhamel's formula, we write (3.1) as

$$
v(t)=U(t, s) v_{s}-i \lambda \int_{s}^{t} U(t, \tau)\left(|x|^{-\gamma} *|v|^{2} v\right)(\tau) d \tau=: \Phi^{s}(v)(t),
$$

where we have dropped the dependence of $U^{\varepsilon}$ upon $\varepsilon$ in the notation, since we assume $\varepsilon=1$ here. Introduce the space

$$
\begin{gathered}
Y_{s, T}=\left\{\phi \in C\left(I_{s, T} ; L^{2}\left(\mathbf{R}^{d}\right)\right):\|\phi\|_{L^{\infty}\left(I_{s, T} ; L^{2}\left(\mathbf{R}^{d}\right)\right)} \leqslant 2\left\|v_{s}\right\|_{L^{2}\left(\mathbf{R}^{d}\right)},\right. \\
\left.\|\phi\|_{L^{8 / \gamma}\left(I_{s, T} ; L^{4 d /(2 d-\gamma)}\left(\mathbf{R}^{d}\right)\right)} \leqslant 2 C(8 / \gamma)\left\|v_{s}\right\|_{L^{2}\left(\mathbf{R}^{d}\right)}\right\}
\end{gathered}
$$

and the distance

$$
d\left(\phi_{1}, \phi_{2}\right)=\left\|\phi_{1}-\phi_{2}\right\|_{L^{8 / \gamma}\left(I_{s, T} ; L^{4 d /(2 d-\gamma)}\right)}
$$

where $I_{s, T}=[s, s+T]$ with $s \geqslant 0$ and $T>0$, and $C(8 / \gamma)$ stems from Proposition [3.4. Then $\left(Y_{s, T}, d\right)$ is a Banach space, as remarked in [18] (see also [7]). Hereafter, we denote by

$$
q=\frac{8}{\gamma}, \quad r=\frac{4 d}{2 d-\gamma}, \quad \theta=\frac{8}{4-\gamma},
$$

and $\|\cdot\|_{L^{a}\left(I_{s, T} ; L^{b}\left(\mathbf{R}^{d}\right)\right)}$ by $\|\cdot\|_{L^{a} L^{b}}$ for simplicity. Notice that $(q, r)$ is admissible and

$$
\frac{1}{q^{\prime}}=\frac{4-\gamma}{4}+\frac{1}{q}=\frac{1}{2}+\frac{1}{\theta} \quad ; \quad \frac{1}{r^{\prime}}=\frac{\gamma}{2 d}+\frac{1}{r} \quad ; \quad \frac{1}{2}=\frac{1}{\theta}+\frac{1}{q} .
$$


By using Strichartz estimates, Hölder inequality and Hardy-Littlewood-Sobolev inequality, we have, for $(\underline{q}, \underline{r}) \in\{(q, r),(\infty, 2)\}$ :

$$
\begin{aligned}
\left\|\Phi^{s}(v)\right\|_{L^{\underline{q}} L^{\underline{r}}} & \leqslant C(\underline{q})\left\|v_{s}\right\|_{L^{2}}+C(\underline{q}, q)\left\||y|^{-\gamma} *|v|^{2} v\right\|_{L^{q^{\prime} L^{r^{\prime}}}} \\
& \leqslant C(\underline{q})\left\|v_{s}\right\|_{L^{2}}+C(\underline{q}, q)\left\||y|^{-\gamma} *|v|^{2}\right\|_{L^{4 /(4-\gamma)} L^{2 d / \gamma}}\|v\|_{L^{q} L^{r}} \\
& \leqslant C(\underline{q})\left\|v_{s}\right\|_{L^{2}}+C\|v\|_{L^{\theta} L^{r}}^{2}\|v\|_{L^{q} L^{r}} \\
& \leqslant C(\underline{q})\left\|v_{s}\right\|_{L^{2}}+C T^{1-\gamma / 2}\|v\|_{L^{q} L^{r}}^{3},
\end{aligned}
$$

for any $v \in Y_{s, T}$, with $C(\infty)=1$ by the standard energy estimate. To show the contraction property of $\Phi^{s}$, for any $v, w \in Y_{s, T}$, we get

$$
\begin{aligned}
\left\|\Phi^{s}(v)-\Phi^{s}(w)\right\|_{L^{q} L^{r}} \lesssim & \left\||y|^{-\gamma} *|v|^{2}\right\|_{L^{4 /(4-\gamma)} L^{2 d / \gamma}}\|v-w\|_{L^{q} L^{r}} \\
& +\left.\left\||y|^{-\gamma} *\right\| v\right|^{2}-|w|^{2} \mid\left\|_{L^{2} L^{2 d / \gamma}}\right\| w \|_{L^{\theta} L^{r}} \\
\lesssim & \leqslant\left(\|v\|_{L^{\theta} L^{r}}^{2}+\|w\|_{L^{\theta} L^{r}}^{2}\right)\|v-w\|_{L^{q} L^{r}} \\
\leqslant & T^{1-\gamma / 2}\left(\|v\|_{L^{q} L^{r}}^{2}+\|w\|_{L^{q} L^{r}}^{2}\right)\|v-w\|_{L^{q} L^{r}} .
\end{aligned}
$$

Thus $\Phi^{s}$ is a contraction from $Y_{s, T}$ to $Y_{s, T}$ provided that $T$ is sufficiently small. Then there exists a unique $v \in Y_{s, T}$ solving (3.1). The global existence of the solution for (3.1) follows from the conservation of $L^{2}$-norm of $v$.

3.3. Growth of higher order Sobolev norms and momenta. We now consider (1.10), that is

$$
i \partial_{t} u+\frac{1}{2} \Delta u=\frac{1}{2}\langle y, Q(t) y\rangle u+\lambda|y|^{-\gamma} *|u|^{2} u \quad ;\left.\quad u\right|_{t=0}=a,
$$

where $Q(t)=\nabla^{2} V(t, x(t))$, so $Q \in C\left(\mathbf{R}_{+}, \mathbf{R}\right)$ is locally Lipschitzean, and bounded, by Assumption 1.1. The second part of Proposition 3.1 follows from the following lemma.

Lemma 3.6. Let $\lambda \in \mathbf{R}$ and $0<\gamma<\min (2, d)$. Suppose $a \in \Sigma^{k}$ for some $k \in \mathbf{N}$. Then there exists a unique $u \in C\left(\mathbf{R}_{+} ; \Sigma^{k}\right)$ solving (1.10), and there exists $C=C(k)$ such that for every admissible pair $\left(q_{1}, r_{1}\right)$,

$$
\left\|y^{\alpha} \partial_{y}^{\beta} u(t)\right\|_{L^{q_{1}}\left([0, t] ; L^{r_{1}}\left(\mathbf{R}^{d}\right)\right)} \leqslant C e^{C t}, \quad \forall t \geqslant 0, \alpha, \beta \in \mathbf{N}^{d},|\alpha|+|\beta| \leqslant k .
$$

Proof. We just state the proof of (3.3), by borrowing the approach in [5]. Applying similar arguments as the proof of Lemma 3.5 and induction, one can prove global existence and uniqueness of the $\Sigma^{k}$ solution for (1.10).

Step 1: $k=0$. For all $t \geqslant 0$ and $\tau>0$, set $I=[t, t+\tau]$. Resuming the computations as in (3.2), we have

$$
\|u\|_{L^{q}\left(I ; L^{r}\left(\mathbf{R}^{d}\right)\right) \cap L^{\infty}\left(I ; L^{2}\left(\mathbf{R}^{d}\right)\right)} \leqslant C\|u(t)\|_{L^{2}}+C_{1} \tau^{1-\gamma / 2}\|u\|_{L^{q}\left(I ; L^{r}\left(\mathbf{R}^{d}\right)\right)}^{3},
$$

where $C$ and $C_{1}$ is independent of $t$ and $\tau$. Then (3.3) for $k=0$ follows from the following bootstrap argument.

Lemma 3.7 (Bootstrap argument). Let $g=g(t)$ be a nonnegative continuous function on $[0, T]$ such that for every $t \in[0, T]$,

$$
g(t) \leqslant M+\delta g(t)^{\kappa}
$$

where $M, \delta$ and $\kappa>1$ are constants such that

$$
M<\left(1-\frac{1}{\kappa}\right) \frac{1}{(\kappa \delta)^{1 /(\kappa-1)}} ; \quad g(0) \leqslant \frac{1}{(\kappa \delta)^{1 /(\kappa-1)}} .
$$


Then

$$
g(t) \leqslant \frac{\kappa}{\kappa-1} M, \quad \forall t \in[0, T] .
$$

For fixed $\tau$ small enough, by the conservation of $L^{2}$ norm of $u$, we choose $\kappa=3$ and $\delta=C_{1} \tau^{1-\gamma / 2}$. Since $0<\gamma<2$, at every increment of time of length of $\tau$, the $L^{q} L^{r}$ and $L^{\infty} L^{2}$ norms of $u$ are bounded by $\frac{3}{2} C\left\|u_{0}\right\|_{L^{2}}$, and (3.3) follows in the case $k=0,\left(q_{1}, r_{1}\right)=(q, r)$. Using Strichartz inequalities again, we have, for any admissible pair $\left(q_{1}, r_{1}\right)$,

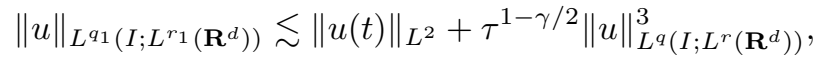

and (3.3) follows in the case $k=0$.

Step 2: Suppose Lemma 3.6 holds for $k-1(k \geqslant 1)$. We denote by $w_{\ell}$ the family of combination of $\alpha$ momenta and $\beta$ space derivatives of $u$ with $|\alpha|+|\beta|=\ell$. Applying $y^{\alpha} \partial_{y}^{\beta}$ to (1.10) formally, we obtain

$$
\begin{aligned}
i \partial_{t} w_{k}+\frac{1}{2} \Delta w_{k}= & \frac{1}{2}\langle y, Q(t) y\rangle w_{k}+\mathrm{v}\left(u, w_{k}\right)+L_{k}(u) \\
& +\lambda \sum_{\substack{0 \leqslant j_{1}, j_{2}, j_{j} \leqslant k-1 \\
j_{1}+j_{2}+j_{3}=k}} c_{j_{1}, j_{2}, j_{3}}|y|^{-\gamma} *\left(w_{j_{1}} \bar{w}_{j_{2}}\right) w_{j_{3}},
\end{aligned}
$$

where

$$
\begin{aligned}
& \mathrm{V}\left(u, w_{k}\right)=|y|^{-\gamma} *\left(w_{k} \bar{u}\right) u+|y|^{-\gamma} *\left(\bar{w}_{k} u\right) u+|y|^{-\gamma} *|u|^{2} w_{k}, \\
& L_{k}(u)=\frac{1}{2} \Omega(t)\left[y^{\alpha} \partial_{y}^{\beta},|y|^{2}\right] u+\frac{1}{2}\left[\Delta, y^{\alpha} \partial_{y}^{\beta}\right] u .
\end{aligned}
$$

Notice that $L_{k}(u)$ is controlled pointwise by $w_{k}$. Still by Strichartz estimates, induction and Step 1, we have

$$
\begin{aligned}
\left\|w_{k}\right\|_{L^{q}\left(I ; L^{r}\right) \cap L^{\infty}\left(I ; L^{2}\right)} \lesssim & \left\|w_{k}(t)\right\|_{L^{2}}+\tau^{1-\frac{\gamma}{2}}\|u\|_{L^{q}\left(I ; L^{r}\right)}\left\|w_{k}\right\|_{L^{q}\left(I ; L^{r}\right)} \\
& +C_{2}\left\|w_{k}\right\|_{L^{1}\left(I, L^{2}\right)}+C_{3} e^{3 C(t+\tau)} \\
\leqslant & C\left\|w_{k}(t)\right\|_{L^{2}}+C_{1} \tau^{1-\frac{\gamma}{2}}\left\|w_{k}\right\|_{L^{q}\left(I ; L^{r}\right)} \\
& +C_{2}\left\|w_{k}\right\|_{L^{1}\left(I, L^{2}\right)}+C_{3} e^{3 C(t+\tau)},
\end{aligned}
$$

where $C, C_{1}, C_{2}$ and $C_{3}$ are independent of $t$ and $\tau$. Choosing $\tau<1$ fixed small enough, the second term on the right hand side of the above inequality can be absorbed by the left hand side.

For any time interval $[0, t]$, split it into finitely many pieces such that the length of every piece at most $\tau$, then we have

$$
\left\|w_{k}\right\|_{L^{q}\left([0, t] ; L^{r}\left(\mathbf{R}^{d}\right)\right) \cap L^{\infty}\left([0, t] ; L^{2}\left(\mathbf{R}^{d}\right)\right)} \lesssim\left\|w_{k}\right\|_{L^{1}\left([0, t] ; L^{2}\left(\mathbf{R}^{d}\right)\right)}+\int_{0}^{t} e^{3 C s} d s .
$$

Lemma 3.6 follows from the Gronwall lemma in the case $\left(q_{1}, r_{1}\right)=(\infty, 2)$. Using Strichartz inequalities again, the general case follows.

\section{Bounded time interval For the CRITICAL CASE}

In this section, we consider the critical case for (1.3) with homogeneous nonlinearity in bounded time interval, and establish a good approximation to the wave function. First, we recall the following lemma from $[6]$. 
Lemma 4.1. Suppose $V$ satisfies Assumption 1.1. Let $(x(t), \xi(t))$ be defined by the trajectories (1.4) and $S(t)$ be the classical action (1.6). Assume $A^{\varepsilon}$ and $B^{\varepsilon}$ are defined as follows:

$$
\begin{aligned}
& A^{\varepsilon}=\sqrt{\varepsilon} \nabla-i \frac{\xi(t)}{\sqrt{\varepsilon}}=\sqrt{\varepsilon} e^{i(S(t)+\xi(t) \cdot(x-x(t))) / \varepsilon} \nabla\left(e^{-i(S(t)+\xi(t) \cdot(x-x(t))) / \varepsilon} \cdot\right) ; \\
& B^{\varepsilon}=\frac{x-x(t)}{\sqrt{\varepsilon}} .
\end{aligned}
$$

Then $A^{\varepsilon}$ and $B^{\varepsilon}$ satisfy the commutation relations:

$$
\begin{aligned}
& {\left[i \varepsilon \partial_{t}+\frac{\varepsilon^{2}}{2} \Delta-V, \quad A^{\varepsilon}\right]=\sqrt{\varepsilon}(\nabla V(t, x)-\nabla V(t, x(t))) ;} \\
& {\left[i \varepsilon \partial_{t}+\frac{\varepsilon^{2}}{2} \Delta-V, \quad B^{\varepsilon}\right]=\varepsilon A^{\varepsilon} .}
\end{aligned}
$$

Proposition 4.2. Under the assumptions in Theorem 1.7, for all $T>0$ which is independent of $\varepsilon>0$, we have

$$
\sup _{0 \leqslant t \leqslant T}\left\|\psi^{\varepsilon}(t)-\varphi^{\varepsilon}(t)\right\|_{\mathcal{H}}=\mathcal{O}(\sqrt{\varepsilon}) .
$$

Proof. Set $w^{\varepsilon}=\psi^{\varepsilon}-\varphi^{\varepsilon}$ : it satisfies

$$
i \varepsilon \partial_{t} w^{\varepsilon}+\frac{\varepsilon^{2}}{2} \Delta w^{\varepsilon}=V w^{\varepsilon}-L^{\varepsilon}+N^{\varepsilon} \quad ;\left.\quad w^{\varepsilon}\right|_{t=0}=0 .
$$

We have denoted by

$$
\begin{aligned}
& L^{\varepsilon}=\left(V(t, x)-T_{2}(t, x, x(t))\right) \varphi^{\varepsilon}, \\
& N^{\varepsilon}=\lambda \varepsilon^{\alpha_{c}}\left(|x|^{-\gamma} *\left|\psi^{\varepsilon}\right|^{2} \psi^{\varepsilon}-|x|^{-\gamma} *\left|\varphi^{\varepsilon}\right|^{2} \varphi^{\varepsilon}\right),
\end{aligned}
$$

where $T_{2}$ corresponds to a second order Taylor approximation:

$$
\begin{aligned}
T_{2}(t, x, x(t))= & V(t, x(t))+\langle\nabla V(t, x(t)), x-x(t)\rangle \\
& +\frac{1}{2}\left\langle x-x(t), \nabla^{2} V(t, x(t))(x-x(t))\right\rangle .
\end{aligned}
$$

By Duhamel's formula, using scaled Strichartz estimates and similar arguments as in the proof of Lemma 3.5, we have

$$
\begin{aligned}
\left\|w^{\varepsilon}\right\|_{L^{q} L^{r}} \lesssim & \varepsilon^{-1 / q}\left\|w^{\varepsilon}(t)\right\|_{L^{2}}+\varepsilon^{-1-1 / q}\left\|L^{\varepsilon}\right\|_{L^{1} L^{2}}+\varepsilon^{-1-2 / q}\left\|N^{\varepsilon}\right\|_{L^{q^{\prime}} L^{r^{\prime}}} \\
\lesssim & \varepsilon^{-1 / q}\left\|w^{\varepsilon}(t)\right\|_{L^{2}}+\varepsilon^{-1-1 / q}\left\|L^{\varepsilon}\right\|_{L^{1} L^{2}} \\
& +\varepsilon^{\alpha_{c}-1-2 / q}\left(\left\|\psi^{\varepsilon}\right\|_{L^{\theta} L^{r}}^{2}+\left\|\varphi^{\varepsilon}\right\|_{L^{\theta} L^{r}}^{2}\right)\left\|w^{\varepsilon}\right\|_{L^{q} L^{r}} .
\end{aligned}
$$

We have used the notation $L^{a} L^{b}$ for $L^{a}\left([t, t+\tau] ; L^{b}\left(\mathbf{R}^{d}\right)\right)$ with $t \geqslant 0$ and $\tau>0$. For all $T>0$, using similar arguments as in the proof of Lemma 3.5. we know $u^{\varepsilon}$, $u \in C\left(\mathbf{R}_{+} ; \Sigma\right)$, then

$$
\|P u\|_{L^{\infty}\left([0, T] ; L^{2}\left(\mathbf{R}^{d}\right)\right)}+\left\|P u^{\varepsilon}\right\|_{L^{\infty}\left([0, T] ; L^{2}\left(\mathbf{R}^{d}\right)\right)} \leqslant C(T), \quad \forall P \in\{\operatorname{Id}, \nabla, x\} .
$$

By the definitions of $A^{\varepsilon}$ and $B^{\varepsilon}$, it is easy to check

$$
\left\|P^{\varepsilon} \varphi^{\varepsilon}\right\|_{L^{\infty}\left([0, T] ; L^{2}\left(\mathbf{R}^{d}\right)\right)}+\left\|P^{\varepsilon} \psi^{\varepsilon}\right\|_{L^{\infty}\left([0, T] ; L^{2}\left(\mathbf{R}^{d}\right)\right)} \leqslant C(T), \quad \forall P^{\varepsilon} \in\left\{\operatorname{Id}, A^{\varepsilon}, B^{\varepsilon}\right\},
$$

where $C(T)$ is independent of $\varepsilon$. Gagliardo-Nirenberg inequality then yields

$$
\left\|\varphi^{\varepsilon}(t)\right\|_{L^{r}\left(\mathbf{R}^{d}\right)} \lesssim \varepsilon^{-\gamma / 8}\left\|\varphi^{\varepsilon}\right\|_{L^{2}\left(\mathbf{R}^{d}\right)}^{1-\gamma / 4}\left\|A^{\varepsilon} \varphi^{\varepsilon}\right\|_{L^{2}\left(\mathbf{R}^{d}\right)}^{\gamma / 4} \leqslant C(T) \varepsilon^{-\gamma / 8}, \quad \forall t \in[0, T] .
$$


Similarly, $\left\|\psi^{\varepsilon}(t)\right\|_{L^{r}}$ is bounded by $C(T) \varepsilon^{-\gamma / 8}$ for all $t \in[0, T]$, where $C(T)$ is independent of $\varepsilon$. Let $[t, t+\tau] \subset[0, T]$, we can rewrite (4.2) as

$$
\begin{aligned}
& \left\|w^{\varepsilon}\right\|_{L^{q} L^{r}} \lesssim \varepsilon^{-1 / q}\left\|w^{\varepsilon}(t)\right\|_{L^{2}}+\varepsilon^{-1-1 / q}\left\|L^{\varepsilon}\right\|_{L^{1} L^{2}} \\
& \quad+\varepsilon^{\alpha_{c}-1-2 / q} \tau^{1-\gamma / 4}\left(\left\|\psi^{\varepsilon}\right\|_{L^{\infty} L^{r}}^{2}+\left\|\varphi^{\varepsilon}\right\|_{L^{\infty} L^{r}}^{2}\right)\left\|w^{\varepsilon}\right\|_{L^{q} L^{r}} \\
& \lesssim \varepsilon^{-1 / q}\left\|w^{\varepsilon}(t)\right\|_{L^{2}}+\varepsilon^{-1-1 / q}\left\|L^{\varepsilon}\right\|_{L^{1} L^{2}}+\varepsilon^{\alpha_{c}-1-2 / q^{-\gamma / 4}} \tau^{1-\gamma / 4}\left\|w^{\varepsilon}\right\|_{L^{q} L^{r}} \\
& \lesssim \varepsilon^{-1 / q}\left\|w^{\varepsilon}(t)\right\|_{L^{2}}+\varepsilon^{-1-1 / q}\left\|L^{\varepsilon}\right\|_{L^{1} L^{2}}+\tau^{1-\gamma / 4}\left\|w^{\varepsilon}\right\|_{L^{q} L^{r}} .
\end{aligned}
$$

Choosing $\tau$ sufficiently small, the last term on the right hand side in (4.3) can be absorbed by the left hand side. Splitting $[0, T]$ into finitely many such intervals, we have

$$
\left\|w^{\varepsilon}\right\|_{L^{q}\left([0, T] ; L^{r}\left(\mathbf{R}^{d}\right)\right)} \lesssim \varepsilon^{-1 / q}\left\|w^{\varepsilon}\right\|_{L^{1}\left([0, T] ; L^{2}\left(\mathbf{R}^{d}\right)\right)}+\varepsilon^{-1-1 / q}\left\|L^{\varepsilon}\right\|_{L^{1}\left([0, T] ; L^{2}\left(\mathbf{R}^{d}\right)\right)} .
$$

Using Strichartz estimates again and resuming the above computations, we get

$$
\left\|w^{\varepsilon}\right\|_{L^{\infty}\left([0, t] ; L^{2}\left(\mathbf{R}^{d}\right)\right)} \lesssim\left\|w^{\varepsilon}\right\|_{L^{1}\left([0, t] ; L^{2}\left(\mathbf{R}^{d}\right)\right)}+\varepsilon^{-1}\left\|L^{\varepsilon}\right\|_{L^{1}\left([0, t] ; L^{2}\left(\mathbf{R}^{d}\right)\right)}, \quad \forall t \in[0, T] .
$$

Thanks to Assumption 1.1 and Proposition 3.1, we have

$$
\left\|L^{\varepsilon}\right\|_{L^{1}\left([0, t] ; L^{2}\left(\mathbf{R}^{d}\right)\right)} \lesssim \varepsilon^{3 / 2}\left\|\langle y\rangle^{3} u\right\|_{L^{1}\left([0, t] ; L^{2}\left(\mathbf{R}^{d}\right)\right)} \lesssim \varepsilon^{3 / 2} e^{C_{0} t},
$$

for any $t \geqslant 0$. Then the Gronwall inequality yields

$$
\left\|w^{\varepsilon}\right\|_{L^{\infty}\left([0, T] ; L^{2}\left(\mathbf{R}^{d}\right)\right)} \leqslant C(T) \sqrt{\varepsilon},
$$

where $C(T)$ is independent of $\varepsilon$.

To establish the control of $\mathcal{H}$ norm, in view of Lemma 4.1 we obtain

$$
\left(i \varepsilon \partial_{t}+\frac{\varepsilon^{2}}{2} \Delta-V\right)\left(A^{\varepsilon} w^{\varepsilon}\right)=\sqrt{\varepsilon}(\nabla V(t, x)-\nabla V(t, x(t))) w^{\varepsilon}-A^{\varepsilon} L^{\varepsilon}+A^{\varepsilon} N^{\varepsilon} .
$$

Using Duhamel's formula and scaled Strichartz estimates again, we lead to

$$
\begin{aligned}
& \left\|A^{\varepsilon} w^{\varepsilon}\right\|_{L^{q} L^{r}} \lesssim \varepsilon^{-1 / q}\left\|A^{\varepsilon} w^{\varepsilon}(t)\right\|_{L^{2}}+\varepsilon^{-1-1 / q}\left\|A^{\varepsilon} L^{\varepsilon}\right\|_{L^{1} L^{2}} \\
& +\varepsilon^{-1-2 / q}\left\|A^{\varepsilon} N^{\varepsilon}\right\|_{L^{q^{\prime}} L^{r^{\prime}}}+\varepsilon^{-1-1 / q}\left\|\sqrt{\varepsilon}(\nabla V(t, x)-\nabla V(t, x(t))) w^{\varepsilon}\right\|_{L^{1} L^{2}} .
\end{aligned}
$$

Note that we have the pointwise estimate:

$$
\left|\sqrt{\varepsilon}(\nabla V(t, x)-\nabla V(t, x(t))) w^{\varepsilon}\right| \leqslant C \varepsilon\left|B^{\varepsilon} w^{\varepsilon}\right|,
$$

where $C$ is independent of $t, x$ and $\varepsilon$. We also have

$$
\left\|A^{\varepsilon} L^{\varepsilon}\right\|_{L^{2}\left(\mathbf{R}^{d}\right)} \lesssim \varepsilon^{3 / 2}\left(\left\|\langle y\rangle^{2} u\right\|_{L^{2}\left(\mathbf{R}^{d}\right)}+\left\|\langle y\rangle^{3} \nabla u\right\|_{L^{2}\left(\mathbf{R}^{d}\right)}\right) .
$$

Having in mind Proposition 3.1, we infer

$$
\left\|A^{\varepsilon} L^{\varepsilon}\right\|_{L^{1}\left([0, t] ; L^{2}\left(\mathbf{R}^{d}\right)\right)} \lesssim \varepsilon^{3 / 2} e^{C_{0} t}, \quad \forall t \geqslant 0 .
$$

We observe that $A^{\varepsilon}$ acts on gauge invariant nonlinearities like a derivative:

$$
A^{\varepsilon}\left(|x|^{-\gamma} *|\phi|^{2} \phi\right)=2 \operatorname{Re}\left(|x|^{-\gamma} *\left(\bar{\phi} A^{\varepsilon} \phi\right)\right) \phi+|x|^{-\gamma} *|\phi|^{2}\left(A^{\varepsilon} \phi\right) .
$$

Then we have

$$
\begin{aligned}
\left\|A^{\varepsilon} N^{\varepsilon}\right\|_{L^{q^{\prime}} L^{r^{\prime}}} \lesssim \varepsilon^{\alpha_{c}}\left\||x|^{-\gamma} *\left(\overline{\psi^{\varepsilon}} A^{\varepsilon} \psi^{\varepsilon}\right) \psi^{\varepsilon}-|x|^{-\gamma} *\left(\overline{\varphi^{\varepsilon}} A^{\varepsilon} \varphi^{\varepsilon}\right) \varphi^{\varepsilon}\right\|_{L^{q^{\prime}} L^{r^{\prime}}} \\
+\varepsilon^{\alpha_{c}}\left\|\left(|x|^{-\gamma} *\left|\psi^{\varepsilon}\right|^{2}\right) A^{\varepsilon} \psi^{\varepsilon}-\left(|x|^{-\gamma} *\left|\varphi^{\varepsilon}\right|^{2}\right) A^{\varepsilon} \varphi^{\varepsilon}\right\|_{L^{q^{\prime}} L^{r^{\prime}}}=: \varepsilon^{\alpha_{c}}(I+I I) .
\end{aligned}
$$


In view of triangle inequality, Hölder inequality and Hardy-Littlewood-Sobolev inequality, by similar arguments as in the proof of Lemma 3.5, we get

$$
\begin{aligned}
I \lesssim & \left\|A^{\varepsilon} w^{\varepsilon}\right\|_{L^{q} L^{r}}\left\|\psi^{\varepsilon}\right\|_{L^{\theta} L^{r}}^{2}+\left\|w^{\varepsilon}\right\|_{L^{q} L^{r}}\left\|A^{\varepsilon} \varphi^{\varepsilon}\right\|_{L^{\theta} L^{r}}\left(\left\|\psi^{\varepsilon}\right\|_{L^{\theta} L^{r}}+\left\|\varphi^{\varepsilon}\right\|_{L^{\theta} L^{r}}\right) \\
& \lesssim\left(\left\|w^{\varepsilon}\right\|_{L^{\theta} L^{r}}^{2}+\left\|\varphi^{\varepsilon}\right\|_{L^{\theta} L^{r}}^{2}\right)\left\|A^{\varepsilon} w^{\varepsilon}\right\|_{L^{q} L^{r}} \\
& +\left(\left\|A^{\varepsilon} \varphi^{\varepsilon}\right\|_{L^{\theta} L^{r}}^{2}+\left\|w^{\varepsilon}\right\|_{L^{\theta} L^{r}}^{2}+\left\|\varphi^{\varepsilon}\right\|_{L^{\theta} L^{r}}^{2}\right)\left\|w^{\varepsilon}\right\|_{L^{q} L^{r}} .
\end{aligned}
$$

The term II satisfies the same estimate. Applying Gagliardo-Nirenberg inequality,

$$
\begin{aligned}
\left\|A^{\varepsilon} \varphi^{\varepsilon}(t)\right\|_{L^{r}\left(\mathbf{R}^{d}\right)} & \lesssim \varepsilon^{-\gamma / 8}\left\|A^{\varepsilon} \varphi^{\varepsilon}\right\|_{L^{2}\left(\mathbf{R}^{d}\right)}^{1-\gamma / 4}\left\|\left(A^{\varepsilon}\right)^{2} \varphi^{\varepsilon}\right\|_{L^{2}\left(\mathbf{R}^{d}\right)}^{\gamma / 4} \\
& \lesssim \varepsilon^{-\gamma / 8}\|\nabla u\|_{L^{2}\left(\mathbf{R}^{d}\right)}^{1-\gamma / 4}\left\|\nabla^{2} u\right\|_{L^{2}\left(\mathbf{R}^{d}\right)}^{\gamma / 4} \leqslant C(T) \varepsilon^{-\gamma / 8}, \quad \forall t \in[0, T],
\end{aligned}
$$

which implies that

$$
\left\|A^{\varepsilon} N^{\varepsilon}\right\|_{L^{q^{\prime}} L^{r^{\prime}}} \lesssim \varepsilon^{\alpha_{c}-\gamma / 4} \tau^{1-\gamma / 4}\left(\left\|A^{\varepsilon} w^{\varepsilon}\right\|_{L^{q} L^{r}}+\left\|w^{\varepsilon}\right\|_{L^{q} L^{r}}\right) .
$$

Then (4.5) can be estimated as

$$
\begin{aligned}
\left\|A^{\varepsilon} w^{\varepsilon}\right\|_{L^{q} L^{r}} & \lesssim \varepsilon^{-1 / q}\left\|A^{\varepsilon} w^{\varepsilon}(t)\right\|_{L^{2}}+\varepsilon^{-1 / q}\left\|B^{\varepsilon} w^{\varepsilon}\right\|_{L^{1} L^{2}} \\
& +\varepsilon^{-1-1 / q}\left\|A^{\varepsilon} L^{\varepsilon}\right\|_{L^{1} L^{2}}+\tau^{1-\gamma / 4}\left(\left\|A^{\varepsilon} w^{\varepsilon}\right\|_{L^{q} L^{r}}+\left\|w^{\varepsilon}\right\|_{L^{q} L^{r}}\right) .
\end{aligned}
$$

Recalling Lemma 4.1, we have

$$
\left(i \varepsilon \partial_{t}+\frac{\varepsilon^{2}}{2} \Delta-V\right)\left(B^{\varepsilon} w^{\varepsilon}\right)=\varepsilon A^{\varepsilon} w^{\varepsilon}-B^{\varepsilon} L^{\varepsilon}+B^{\varepsilon} N^{\varepsilon} .
$$

Proceeding like above, we come up with

$$
\begin{aligned}
\left\|B^{\varepsilon} w^{\varepsilon}\right\|_{L^{q} L^{r}} \lesssim & \varepsilon^{-1 / q}\left\|B^{\varepsilon} w^{\varepsilon}(t)\right\|_{L^{2}}+\varepsilon^{-1 / q}\left\|A^{\varepsilon} w^{\varepsilon}\right\|_{L^{1} L^{2}} \\
& +\varepsilon^{-1-1 / q}\left\|B^{\varepsilon} L^{\varepsilon}\right\|_{L^{1} L^{2}}+\tau^{1-\gamma / 4}\left(\left\|B^{\varepsilon} w^{\varepsilon}\right\|_{L^{q} L^{r}}+\left\|w^{\varepsilon}\right\|_{L^{q} L^{r}}\right) .
\end{aligned}
$$

Summing over (4.3), (4.7) and (4.8), we have

$$
\begin{aligned}
& \sum_{P^{\varepsilon} \in\left\{\operatorname{Id}, A^{\varepsilon}, B^{\varepsilon}\right\}}\left\|P^{\varepsilon} w^{\varepsilon}\right\|_{L^{q} L^{r}} \lesssim \varepsilon^{-1 / q} \sum_{P^{\varepsilon} \in\left\{\operatorname{Id}, A^{\varepsilon}, B^{\varepsilon}\right\}}\left(\left\|P^{\varepsilon} w^{\varepsilon}(t)\right\|_{L^{2}}+\left\|P^{\varepsilon} w^{\varepsilon}\right\|_{L^{1} L^{2}}\right) \\
& +\varepsilon^{-1-1 / q} \sum_{P^{\varepsilon} \in\left\{\operatorname{Id}, A^{\varepsilon}, B^{\varepsilon}\right\}}\left\|P^{\varepsilon} L^{\varepsilon}\right\|_{L^{1} L^{2}}+\tau^{1-\gamma / 4} \sum_{P^{\varepsilon} \in\left\{\operatorname{Id}, A^{\varepsilon}, B^{\varepsilon}\right\}}\left\|P^{\varepsilon} w^{\varepsilon}\right\|_{L^{q} L^{r}} .
\end{aligned}
$$

Take $\tau$ sufficiently small such that the last term of the right hand side in the above inequality can be absorbed by the left hand side. Using scaled Strichartz estimates again, resuming the above computations, for any fixed $T>0$ and any $t \in[0, T]$,

$$
\begin{aligned}
\sum_{P^{\varepsilon} \in\left\{\operatorname{Id}, A^{\varepsilon}, B^{\varepsilon}\right\}}\left\|P^{\varepsilon} w^{\varepsilon}\right\|_{L^{\infty}\left([0, t] ; L^{2}\left(\mathbf{R}^{d}\right)\right)} & \sum_{P^{\varepsilon} \in\left\{\mathrm{Id}, A^{\varepsilon}, B^{\varepsilon}\right\}}\left\|P^{\varepsilon} w^{\varepsilon}\right\|_{L^{1}\left([0, t] ; L^{2}\left(\mathbf{R}^{d}\right)\right)} \\
& +\varepsilon^{-1} \sum_{P^{\varepsilon} \in\left\{\mathrm{Id}, A^{\varepsilon}, B^{\varepsilon}\right\}}\left\|P^{\varepsilon} L^{\varepsilon}\right\|_{L^{1}\left([0, t] ; L^{2}\left(\mathbf{R}^{d}\right)\right)} .
\end{aligned}
$$

We end up with

$$
\left\|w^{\varepsilon}\right\|_{L^{\infty}([0, t] ; \mathcal{H})} \lesssim\left\|w^{\varepsilon}\right\|_{L^{1}([0, t] ; \mathcal{H})}+\sqrt{\varepsilon}, \quad \forall t \in[0, T],
$$

and Proposition 4.2 follows from Gronwall lemma. 


\section{LARGE TIME APPROXIMATION}

In this section, we improve the time of validity of the error estimate proven in \$4 in two cases: the linearizable case $\alpha>\alpha_{c}$, and the non-linearizable case $\alpha=\alpha_{c}$, thus proving Proposition 1.6 and Theorem 1.7.

5.1. Proof of Proposition 1.6. Set $w^{\varepsilon}=\psi^{\varepsilon}-\varphi_{\text {lin }}^{\varepsilon}$ : it satisfies

$$
i \varepsilon \partial_{t} w^{\varepsilon}+\frac{\varepsilon^{2}}{2} \Delta u=V w^{\varepsilon}-L^{\varepsilon}+N^{\varepsilon} \quad ;\left.\quad w^{\varepsilon}\right|_{t=0}=0,
$$

where

$$
L^{\varepsilon}=\left(V(t, x)-T_{2}(t, x, x(t))\right) \varphi_{\operatorname{lin}}^{\varepsilon} \quad ; \quad N^{\varepsilon}=\lambda \varepsilon^{\alpha}|x|^{-\gamma} *\left|\varphi_{\operatorname{lin}}^{\varepsilon}+w^{\varepsilon}\right|^{2}\left(\varphi_{\operatorname{lin}}^{\varepsilon}+w^{\varepsilon}\right) .
$$

Using scaled Strichartz estimates, we have, for $t>0$,

$$
\begin{aligned}
\left\|w^{\varepsilon}\right\|_{L_{t}^{q} L^{r}} \lesssim & \varepsilon^{-1-1 / q}\left\|L^{\varepsilon}\right\|_{L_{t}^{1} L^{2}}+\varepsilon^{-1-2 / q}\left\|N^{\varepsilon}\right\|_{L_{t}^{q^{\prime}} L^{r^{\prime}}} \\
\lesssim & \varepsilon^{-1-1 / q}\left\|L^{\varepsilon}\right\|_{L_{t}^{1} L^{2}}+\varepsilon^{\alpha-1-2 / q}\left\|\varphi_{\operatorname{lin}}^{\varepsilon}\right\|_{L_{t}^{\theta} L^{r}}^{2}\left\|\varphi_{\operatorname{lin}}^{\varepsilon}\right\|_{L_{t}^{q} L^{r}} \\
& +\varepsilon^{\alpha-1-2 / q}\left(\left\|w^{\varepsilon}\right\|_{L_{t}^{\theta} L^{r}}^{2}+\left\|\varphi_{\operatorname{lin}}^{\varepsilon}\right\|_{L_{t}^{\theta} L^{r}}^{2}\right)\left\|w^{\varepsilon}\right\|_{L_{t}^{q} L^{r}},
\end{aligned}
$$

where $L_{t}^{a} L^{b}$ stands for $L^{a}\left([0, t] ; L^{b}\left(\mathbf{R}^{d}\right)\right)$. In view of Proposition 3.1. GagliardoNirenberg inequality yields

$$
\left\|\varphi_{\operatorname{lin}}^{\varepsilon}(t)\right\|_{L^{r}\left(\mathbf{R}^{d}\right)} \lesssim \varepsilon^{-\gamma / 8}\left\|\varphi_{\operatorname{lin}}^{\varepsilon}\right\|_{L^{2}\left(\mathbf{R}^{d}\right)}^{1-\gamma / 4}\left\|A^{\varepsilon} \varphi_{\operatorname{lin}}^{\varepsilon}\right\|_{L^{2}\left(\mathbf{R}^{d}\right)}^{\gamma / 4} \leqslant C \varepsilon^{-\gamma / 8} e^{C_{0} t}, \quad \forall t \geqslant 0,
$$

where $C$ and $C_{0}$ are independent of $\varepsilon$ and $t$. We use a bootstrap argument, relying upon the estimate

$$
\left\|w^{\varepsilon}(t)\right\|_{L^{r}\left(\mathbf{R}^{d}\right)} \leqslant \varepsilon^{-\gamma / 8} e^{C_{0} t}
$$

for $t \in\left[0, T^{\varepsilon}\right]$, where $T^{\varepsilon}$ may (and will) depend on $\varepsilon$. Since $w^{\varepsilon}$ is expected to be small compared to $\varphi_{\text {lin }}^{\varepsilon}$, such an estimate looks sensible. We come up with

$\left\|w^{\varepsilon}\right\|_{L_{t}^{q} L^{r}} \leqslant C \varepsilon^{-1-1 / q}\left\|L^{\varepsilon}\right\|_{L_{t}^{1} L^{2}}+C \varepsilon^{\alpha-\alpha_{c}} e^{2 C_{0} t}\left\|\varphi_{\text {lin }}^{\varepsilon}\right\|_{L_{t}^{q} L^{r}}+C \varepsilon^{\alpha-\alpha_{c}} e^{2 C_{0} t}\left\|w^{\varepsilon}\right\|_{L_{t}^{q} L^{r}}$.

Now assume $T^{\varepsilon}$ is chosen so that

$$
C \varepsilon^{\alpha-\alpha_{c}} e^{2 C_{0} T^{\varepsilon}} \leqslant \frac{1}{2}
$$

Then we have

$$
\left\|w^{\varepsilon}\right\|_{L_{t}^{q} L^{r}} \leqslant 2 C \varepsilon^{-1-1 / q}\left\|L^{\varepsilon}\right\|_{L_{t}^{1} L^{2}}+2 C \varepsilon^{\alpha-\alpha_{c}} e^{2 C_{0} t}\left\|\varphi_{\operatorname{lin}}^{\varepsilon}\right\|_{L_{t}^{q} L^{r}}
$$

Using scaled Strichartz estimates again, we get

$$
\begin{aligned}
\left\|w^{\varepsilon}\right\|_{L_{t}^{\infty} L^{2}} & \lesssim \varepsilon^{-1}\left\|L^{\varepsilon}\right\|_{L_{t}^{1} L^{2}}+\varepsilon^{\alpha-1-1 / q} e^{2 C_{0} t}\left\|\varphi_{\text {lin }}^{\varepsilon}\right\|_{L_{t}^{q} L^{r}} \\
& \leqslant C\left(\sqrt{\varepsilon} e^{C_{0} t}+\varepsilon^{\alpha-\alpha_{c}} e^{3 C_{0} t}\right) .
\end{aligned}
$$

Apply $A^{\varepsilon}$ and $B^{\varepsilon}$ respectively to Equation (5.1), then by the same arguments as in the proof of Proposition 4.2, we get

$$
\begin{aligned}
& \sum_{P^{\varepsilon} \in\left\{A^{\varepsilon}, B^{\varepsilon}\right\}}\left\|P^{\varepsilon} w^{\varepsilon}\right\|_{L_{t}^{q} L^{r}} \lesssim \varepsilon^{-1 / q} \sum_{P^{\varepsilon} \in\left\{A^{\varepsilon}, B^{\varepsilon}\right\}}\left\|P^{\varepsilon} w^{\varepsilon}\right\|_{L_{t}^{1} L^{2}}+\varepsilon^{\alpha-\alpha_{c}} e^{3 C_{0} t} \\
& +\varepsilon^{-1-1 / q} \sum_{P^{\varepsilon} \in\left\{A^{\varepsilon}, B^{\varepsilon}\right\}}\left\|P^{\varepsilon} L^{\varepsilon}\right\|_{L_{t}^{1} L^{2}}+\varepsilon^{\alpha-\alpha_{c}} e^{2 C_{0} t} \sum_{P^{\varepsilon} \in\left\{\operatorname{Id}, A^{\varepsilon}, B^{\varepsilon}\right\}}\left\|P^{\varepsilon} w^{\varepsilon}\right\|_{L_{t}^{q} L^{r}} .
\end{aligned}
$$


As long as (5.2) holds, we can use the same absorption argument as above to treat the last term. By using scaled Strichartz again, we obtain

$$
\left\|w^{\varepsilon}\right\|_{L^{\infty}([0, t] ; \mathcal{H})} \lesssim\left\|w^{\varepsilon}\right\|_{L^{1}([0, t] ; \mathcal{H})}+\varepsilon^{\alpha-\alpha_{c}} e^{3 C_{0} t}+\varepsilon^{-1}\left\|L^{\varepsilon}\right\|_{L^{1}([0, t] ; \mathcal{H})} .
$$

Since

$$
\varepsilon^{-1}\left\|L^{\varepsilon}\right\|_{L^{1}([0, t] ; \mathcal{H})} \leqslant C \sqrt{\varepsilon} e^{C t},
$$

we end up with

$$
\left\|w^{\varepsilon}\right\|_{L^{\infty}([0, t] ; \mathcal{H})} \leqslant C\left(\left\|w^{\varepsilon}\right\|_{L^{1}([0, t] ; \mathcal{H})}+\varepsilon^{\kappa} e^{C_{1} t}\right),
$$

where $\kappa=\min \left\{\frac{1}{2}, \alpha-\alpha_{c}\right\}$ and $C_{1}$ is independent of $\varepsilon$ and $t$. Gronwall lemma yields

$$
\left\|w^{\varepsilon}(t)\right\|_{\mathcal{H}} \leqslant C \varepsilon^{\kappa} e^{C_{1} t}
$$

so long as (5.2) holds. Gagliardo-Nirenberg inequality yields

$$
\left\|w^{\varepsilon}(t)\right\|_{L^{r}\left(\mathbf{R}^{d}\right)} \lesssim \varepsilon^{-\gamma / 8}\left\|w^{\varepsilon}\right\|_{L^{2}\left(\mathbf{R}^{d}\right)}^{1-\gamma / 4}\left\|A^{\varepsilon} w^{\varepsilon}\right\|_{L^{2}\left(\mathbf{R}^{d}\right)}^{\gamma / 4} \leqslant C \varepsilon^{\kappa-\gamma / 8} e^{C_{1} t} .
$$

Proposition 1.6 then follows, by choosing $T^{\varepsilon}=C_{2} \ln \frac{1}{\varepsilon}$ with $C_{2}>0$ sufficiently small and independent of $\varepsilon$, and $\left.\varepsilon \in] 0, \varepsilon_{0}\right]$ for $\varepsilon_{0}>0$ sufficiently small.

5.2. Proof of Theorem 1.7. We know explain how to upgrade Proposition 4.2 to Theorem 1.7 by examining the large time behavior of the quantities involved in the proof. By Proposition 3.1, we know that $u \in C\left(\mathbf{R}_{+}, \Sigma^{k}\right)$ provided that the initial datum $a \in \Sigma^{k}$. Recalling Step 1 in Lemma 3.6. we showed that for any $t \geqslant 0$ and $\tau>0$ sufficiently small,

$$
\|u\|_{L^{q}\left([t, t+\tau] ; L^{r}\left(\mathbf{R}^{d}\right)\right)} \leqslant \frac{3}{2} C\|u(0, \cdot)\|_{L^{2}\left(\mathbf{R}^{d}\right)}=\frac{3}{2} C\|a\|_{L^{2}\left(\mathbf{R}^{d}\right)} .
$$

where $C$ is independent of $t, \tau$. For any $t \geqslant 0$, fixed $\tau \in] 0,1[$, split $[t, t+1]$ into finitely many pieces with length at most $\tau$, then we obtain

$$
\left\|\varphi^{\varepsilon}\right\|_{L^{q}\left([t, t+1] ; L^{r}\left(\mathbf{R}^{d}\right)\right)}=\varepsilon^{-\gamma / 8}\|u\|_{L^{q}\left([t, t+1] ; L^{r}\left(\mathbf{R}^{d}\right)\right)} \leqslant C \varepsilon^{-\gamma / 8}\|a\|_{L^{2}\left(\mathbf{R}^{d}\right)}, \quad \forall t \geqslant 0 .
$$

Using Proposition 4.2 and Gagliardo-Nirenberg inequality, we know that there exists $\varepsilon_{0}>0$ such that

$$
\left\|w^{\varepsilon}\right\|_{L^{q}\left([t, t+1] ; L^{r}\left(\mathbf{R}^{d}\right)\right)} \leqslant \varepsilon^{-\gamma / 8}\|a\|_{L^{2}\left(\mathbf{R}^{d}\right)},
$$

for $t \in[0,1]$. Suppose that (5.3) holds for $t \in\left[0, T^{\varepsilon}\right]\left(T^{\varepsilon} \geqslant 1\right)$, and let $t, \tau>0$ with $t+\tau \leqslant T^{\varepsilon}$. Denote by $L^{a} L^{b}$ the space $L^{a}\left([t, t+\tau] ; L^{b}\left(\mathbf{R}^{d}\right)\right)$. Scaled Strichartz estimates, Hölder inequality and Hardy-Littlewood-Sobolev inequality yield

$$
\begin{aligned}
\left\|w^{\varepsilon}\right\|_{L^{q} L^{r}} \lesssim & \varepsilon^{-1 / q}\left\|w^{\varepsilon}(t)\right\|_{L^{2}}+\varepsilon^{-1-1 / q}\left\|L^{\varepsilon}\right\|_{L^{1} L^{2}} \\
& +\varepsilon^{\alpha_{c}-1-2 / q}\left(\left\|\varphi^{\varepsilon}\right\|_{L^{\theta} L^{r}}^{2}+\left\|w^{\varepsilon}\right\|_{L^{\theta} L^{r}}^{2}\right)\left\|w^{\varepsilon}\right\|_{L^{q} L^{r}} \\
\leqslant & C\left(\varepsilon^{-1 / q}\left\|w^{\varepsilon}(t)\right\|_{L^{2}}+\varepsilon^{-1-1 / q}\left\|L^{\varepsilon}\right\|_{L^{1} L^{2}}+\tau^{1-\gamma / 2}\left\|w^{\varepsilon}\right\|_{L^{q} L^{r}}\right),
\end{aligned}
$$

where $C$ is independent of $\varepsilon, t, \tau$. Choose $\tau \in] 0,1]$ sufficiently small such that the last term on the above right hand side can be absorbed by the left hand side. Using scaled Strichartz estimates again and resuming the previous computations, we end up with

$$
\left\|w^{\varepsilon}\right\|_{L^{\infty}\left([0, t] ; L^{2}\left(\mathbf{R}^{d}\right)\right)} \leqslant C \int_{0}^{t}\left\|w^{\varepsilon}\right\|_{L^{\infty}\left([0, s] ; L^{2}\left(\mathbf{R}^{d}\right)\right)} d s+C \varepsilon^{-1} \int_{0}^{t}\left\|L^{\varepsilon}(s)\right\|_{L^{2}} d s .
$$


The last term is controlled thanks to Proposition 3.1, and Gronwall lemma yields

$$
\left\|w^{\varepsilon}\right\|_{L^{\infty}\left([0, t] ; L^{2}\left(\mathbf{R}^{d}\right)\right)} \leqslant C \sqrt{\varepsilon} e^{C_{1} t} .
$$

Resuming Strichartz inequalities, and splitting the interval $[t, t+1]$ into finitely many intervals of length $\tau$, the above computation yields:

$$
\left\|w^{\varepsilon}\right\|_{L^{q}\left([t, t+1] ; L^{r}\right)} \lesssim \varepsilon^{-1 / q}\left\|w^{\varepsilon}(t)\right\|_{L^{2}}+\varepsilon^{-1-1 / q}\left\|L^{\varepsilon}\right\|_{L^{1}\left([t, t+1] ; L^{2}\right)} \lesssim \varepsilon^{-\gamma / 8} \sqrt{\varepsilon} e^{C_{1} t} .
$$

Setting $T^{\varepsilon}=C_{2} \ln \frac{1}{\varepsilon}$ with $C_{2}>0$ sufficiently small and independent of $\varepsilon$, and $\left.\varepsilon \in] 0, \varepsilon_{0}\right]$ for $\varepsilon_{0}>0$ sufficiently small, we see that (5.3) holds for all $t \in\left[0, T^{\varepsilon}\right]$; the first part of Theorem 1.7 follows.

As in the proof of Proposition 4.2, we also have

$$
\begin{aligned}
& \sum_{P^{\varepsilon} \in\left\{\operatorname{Id}, A^{\varepsilon}, B^{\varepsilon}\right\}}\left\|P^{\varepsilon} w^{\varepsilon}\right\|_{L^{q} L^{r}} \lesssim \varepsilon^{-1 / q} \sum_{P^{\varepsilon} \in\left\{\operatorname{Id}, A^{\varepsilon}, B^{\varepsilon}\right\}}\left(\left\|P^{\varepsilon} w^{\varepsilon}(t)\right\|_{L^{2}}+\left\|P^{\varepsilon} w^{\varepsilon}\right\|_{L^{1} L^{2}}\right) \\
& +\varepsilon^{1-1 / q} \sum_{P^{\varepsilon} \in\left\{\mathrm{Id}, A^{\varepsilon}, B^{\varepsilon}\right\}}\left\|P^{\varepsilon} L^{\varepsilon}\right\|_{L^{1} L^{2}}+\varepsilon^{\alpha_{c}-1-2 / q}\left\|w^{\varepsilon}\right\|_{L^{q} L^{r}} \sum_{P^{\varepsilon} \in\left\{A^{\varepsilon}, B^{\varepsilon}\right\}}\left\|P^{\varepsilon} \varphi^{\varepsilon}\right\|_{L^{\theta} L^{r}}^{2} \\
& +\varepsilon^{\alpha_{c}-1-2 / q}\left(\left\|w^{\varepsilon}\right\|_{L^{\theta} L^{r}}^{2}+\left\|\varphi^{\varepsilon}\right\|_{L^{\theta} L^{r}}^{2}\right) \sum_{P^{\varepsilon} \in\left\{\operatorname{Id}, A^{\varepsilon}, B^{\varepsilon}\right\}}\left\|P^{\varepsilon} w^{\varepsilon}\right\|_{L^{q} L^{r} .}
\end{aligned}
$$

Choosing $\tau$ sufficiently small, the last term on the right hand side of the above inequality can be absorbed by the left hand side. Notice that so long as (5.3) holds,

$$
\varepsilon^{\alpha_{c}-1-\frac{2}{q}}\left\|w^{\varepsilon}\right\|_{L^{q} L^{r}}\left(\left\|A^{\varepsilon} \varphi^{\varepsilon}\right\|_{L^{\theta} L^{r}}^{2}+\left\|B^{\varepsilon} \varphi^{\varepsilon}\right\|_{L^{\theta} L^{r}}^{2}\right) \lesssim \varepsilon^{-\gamma / 8} e^{2 C_{0} t} .
$$

Using scaled Strichartz estimates again, mimicking the above computations, and using Proposition 3.1, Gronwall lemma yields

$$
\left\|w^{\varepsilon}\right\|_{L^{\infty}([0, t] ; \mathcal{H})} \leqslant C \sqrt{\varepsilon} e^{C_{1} t},
$$

which completes the proof of Theorem 1.7

\section{NONLINEAR SUPERPOSITION}

In this section, we consider the nonlinear superposition for the critical case $\alpha=$ $\alpha_{c}$. The arguments to prove Theorem 1.9 and Theorem 1.10 are quite similar to the proof of Proposition 4.2 and Theorem 1.7, respectively. The important aspect to be understood is the interaction between the two profiles $\varphi_{1}^{\varepsilon}$ and $\varphi_{2}^{\varepsilon}$. The error $w^{\varepsilon}=\psi^{\varepsilon}-\varphi_{1}^{\varepsilon}-\varphi_{2}^{\varepsilon}$ satisfies

$$
i \varepsilon \partial_{t} w^{\varepsilon}+\frac{\varepsilon^{2}}{2} \Delta w^{\varepsilon}=V w^{\varepsilon}-L^{\varepsilon}+N^{\varepsilon} \quad ;\left.\quad w^{\varepsilon}\right|_{t=0}=0,
$$

where

$$
\begin{aligned}
L^{\varepsilon} & =\left(V(t, x)-T_{2}(t, x, x(t))\right)\left(\varphi_{1}^{\varepsilon}+\varphi_{2}^{\varepsilon}\right), \\
N^{\varepsilon} & =\lambda \varepsilon^{\alpha_{c}}\left(F\left(w^{\varepsilon}+\varphi_{1}^{\varepsilon}+\varphi_{2}^{\varepsilon}\right)-F\left(\varphi_{1}^{\varepsilon}\right)-F\left(\varphi_{2}^{\varepsilon}\right)\right), \quad F(\psi):=\left(|x|^{-\gamma} *|\psi|^{2}\right) \psi .
\end{aligned}
$$

As in [6], decompose $N^{\varepsilon}$ into two parts: a semilinear term

$$
N_{S}^{\varepsilon}=\lambda \varepsilon^{\alpha_{c}}\left(F\left(w^{\varepsilon}+\varphi_{1}^{\varepsilon}+\varphi_{2}^{\varepsilon}\right)-F\left(\varphi_{1}^{\varepsilon}+\varphi_{2}^{\varepsilon}\right)\right),
$$

and an interaction (source) term

$$
N_{I}^{\varepsilon}=\lambda \varepsilon^{\alpha_{c}}\left(F\left(\varphi_{1}^{\varepsilon}+\varphi_{2}^{\varepsilon}\right)-F\left(\varphi_{1}^{\varepsilon}\right)-F\left(\varphi_{2}^{\varepsilon}\right)\right) .
$$


As noticed in [6], the term $N_{S}^{\varepsilon}$ can be treated as in the case of a single wave packet: the key point is to estimate

$$
\varepsilon^{\alpha_{c}-1}\left\|N_{I}^{\varepsilon}\right\|_{L^{1}\left([0, t] ; \Sigma_{\varepsilon}\right)}
$$

since $N_{I}^{\varepsilon}$ plays the role of a source term. This is the only new term to control to infer Theorem 1.9 and Theorem 1.10 from Proposition 4.2 and Theorem 1.7.

Lemma 6.1. Let $0<\gamma<\min (2, d), T \geqslant 0$ and $0<\sigma<\frac{1}{2}$. Denote

$$
I^{\varepsilon}(T)=\left\{t \in[0, T] ; \quad\left|x_{1}(t)-x_{2}(t)\right| \leqslant \varepsilon^{\sigma}\right\} .
$$

Then for any $k \in \mathbf{N}$ with $k>\gamma$,

$$
\varepsilon^{-1}\left\|N_{I}^{\varepsilon}\right\|_{L^{1}\left([0, T] ; \Sigma_{\varepsilon}\right)} \lesssim\left(M_{k+2}(T)\right)^{3}\left(T \varepsilon^{\gamma(1 / 2-\sigma)}+\left|I^{\varepsilon}(T)\right|\right) e^{C T},
$$

where $M_{k}(T)=\sup \left\{\left\|u_{j}\right\|_{L^{\infty}\left([0, T] ; \Sigma^{k}\right)} ; j \in\{1,2\}\right\}$.

In particular, if $T>0$ is independent of $\varepsilon$, then

$$
\varepsilon^{-1}\left\|N_{I}^{\varepsilon}\right\|_{L^{1}\left([0, T] ; \Sigma_{\varepsilon}\right)} \lesssim\left(M_{k+2}(T)\right)^{3}\left(\varepsilon^{\gamma(1 / 2-\sigma)}+\varepsilon^{\sigma}\right) .
$$

Proof. We compute

$$
\begin{aligned}
N_{I}^{\varepsilon}= & \varepsilon^{\alpha_{c}}\left(\left(|x|^{-\gamma} *\left|\varphi_{1}^{\varepsilon}\right|^{2}\right) \varphi_{2}^{\varepsilon}+\left(|x|^{-\gamma} *\left|\varphi_{2}^{\varepsilon}\right|^{2}\right) \varphi_{1}^{\varepsilon}\right) \\
& +2 \varepsilon^{\alpha_{c}}\left(|x|^{-\gamma} *\left(\operatorname{Re}\left(\varphi_{1}^{\varepsilon} \overline{\varphi_{2}^{\varepsilon}}\right)\right)\right)\left(\varphi_{1}^{\varepsilon}+\varphi_{2}^{\varepsilon}\right) .
\end{aligned}
$$

On the complement of $I^{\varepsilon}(T)$, we will use Peetre inequality: for $\eta \in \mathbf{R}^{d}$,

$$
\sup _{x \in \mathbf{R}^{d}}\left(\langle x\rangle^{-1}\langle x-\eta\rangle^{-1}\right) \lesssim \frac{1}{\langle\eta\rangle} \lesssim \frac{1}{|\eta|} .
$$

Denote $\eta^{\varepsilon}=\frac{x_{1}(t)-x_{2}(t)}{\sqrt{\varepsilon}}$. For the last term in (6.2), we have, for $j \in\{1,2\}$,

$$
\begin{aligned}
\varepsilon^{\alpha_{c}-1} \int_{[0, T] \backslash I^{\varepsilon}(T)}\left\|\left(|x|^{-\gamma} *\left|\varphi_{1}^{\varepsilon}(t, x) \varphi_{2}^{\varepsilon}(t, x)\right|\right) \varphi_{j}^{\varepsilon}(t, x)\right\|_{L^{2}\left(\mathbf{R}^{d}\right)} d t \\
=\int_{[0, T] \backslash I^{\varepsilon}(T)}\left\|\left(|x|^{-\gamma} *\left|u_{1}(t, x) u_{2}\left(t, x-\eta^{\varepsilon}\right)\right|\right) u_{j}(t, x)\right\|_{L^{2}\left(\mathbf{R}^{d}\right)} d t \\
\lesssim\left\|\left.|| x\right|^{-\gamma} *\left|\langle x\rangle^{k} u_{1}(t, x)\left\langle x-\eta^{\varepsilon}\right\rangle^{k} u_{2}\left(t, x-\eta^{\varepsilon}\right)\right|\right\|_{L^{\infty} L^{2 d / \gamma}}\left\|u_{j}\right\|_{L^{\infty} L^{2 d /(d-\gamma)}} \\
\quad \times \int_{[0, T] \backslash I^{\varepsilon}(T)} \frac{d t}{\left|\eta^{\varepsilon}(t)\right|^{k}} .
\end{aligned}
$$

In view of Hardy-Littlewood-Sobolev inequality and Sobolev embedding, we have:

$$
\begin{aligned}
& \left\||x|^{-\gamma} *\left|\langle x\rangle^{k} u_{1}(t, x)\left\langle x-\eta^{\varepsilon}\right\rangle^{k} u_{2}\left(t, x-\eta^{\varepsilon}\right)\right|\right\|_{L^{\infty} L^{2 d / \gamma}} \\
\lesssim & \left\|\langle x\rangle^{k} u_{1}(x)\right\|_{L^{\infty} L^{4 d /(2 d-\gamma)}}\left\|\left\langle x-\eta^{\varepsilon}\right\rangle^{k} u_{2}\left(x-\eta^{\varepsilon}\right)\right\|_{L^{\infty} L^{4 d /(2 d-\gamma)}} \\
\lesssim & \left\|\langle x\rangle^{k} u_{1}(x)\right\|_{L^{\infty} H^{1}}\left\|\left\langle x-\eta^{\varepsilon}\right\rangle^{k} u_{2}\left(x-\eta^{\varepsilon}\right)\right\|_{L^{\infty} H^{1}} \lesssim\left(M_{k+1}(T)\right)^{2} .
\end{aligned}
$$

On the other hand, we have

$$
\int_{[0, T] \backslash I^{\varepsilon}(T)} \frac{d t}{\left|\eta^{\varepsilon}(t)\right|^{k}}=\int_{[0, T] \backslash I^{\varepsilon}(T)} \frac{\varepsilon^{k / 2}}{\left|x_{1}(t)-x_{2}(t)\right|^{k}} d t \leqslant \varepsilon^{k(1 / 2-\sigma)} T .
$$

It follows that

$$
\varepsilon^{\alpha_{c}-1} \int_{[0, T] \backslash I^{\varepsilon}(T)}\left\|\left(|x|^{-\gamma} *\left|\varphi_{1}^{\varepsilon} \varphi_{2}^{\varepsilon}\right|\right) \varphi_{j}^{\varepsilon}(t, x)\right\|_{L^{2}\left(\mathbf{R}^{d}\right)} d t \lesssim\left(M_{k+1}(T)\right)^{3} \varepsilon^{k(1 / 2-\sigma)} T .
$$


The first two terms in (6.2) are of the same form, so we consider the first one only:

$$
\begin{aligned}
& \varepsilon^{\alpha_{c}-1} \int_{[0, T] \backslash I^{\varepsilon}(T)}\left\|\left(|x|^{-\gamma} *\left|\varphi_{1}^{\varepsilon}\right|^{2}\right) \varphi_{2}^{\varepsilon}\right\|_{L^{2}\left(\mathbf{R}^{d}\right)} d t \\
= & \int_{[0, T] \backslash I^{\varepsilon}(T)}\left\|\left(\int_{\mathbf{R}^{d}}|y|^{-\gamma}\left|u_{1}\left(t, x-y-\eta^{\varepsilon}\right)\right|^{2} d y\right) u_{2}(t, x)\right\|_{L^{2}\left(\mathbf{R}^{d}\right)} d t .
\end{aligned}
$$

In view of Peetre inequality, using similar arguments as above, we have

$$
\begin{aligned}
& \int_{[0, T] \backslash I^{\varepsilon}(T)}\left\|\int_{\left|y+\eta^{\varepsilon}\right|>\left|\eta^{\varepsilon}\right|}|y|^{-\gamma}\left|u_{1}\left(t, x-y-\eta^{\varepsilon}\right)\right|^{2} d y \times u_{2}(t, x)\right\|_{L^{2}\left(\mathbf{R}^{d}\right)} d t \\
\lesssim & \left(\int_{[0, T] \backslash I^{\varepsilon}(T)} \sup _{\substack{x \in \mathbf{R}^{d} \\
\left|y+\eta^{\varepsilon}\right|>\left|\eta^{\varepsilon}\right|}}\left\langle x-y-\eta^{\varepsilon}\right\rangle^{-k}\langle x\rangle^{-k} d t\right)\left\|\langle x\rangle^{k} u_{2}(t, x)\right\|_{L^{\infty} L^{2 d /(d-\gamma)}} \\
& \times\left\|\int|y|^{-\gamma}\left|u_{1}\left(t, x-y-\eta^{\varepsilon}\right)\right|^{2}\left\langle x-y-\eta^{\varepsilon}\right\rangle^{k} d y\right\|_{L^{\infty} L^{2 d / \gamma}} \\
\lesssim & \int_{[0, T] \backslash I^{\varepsilon}(T)} \frac{d t}{\left|\eta^{\varepsilon}\right|}\left\|\langle x\rangle^{k} u_{2}(t, x)\right\|_{L^{\infty} L^{2 d /(d-\gamma)}}\left\|\langle x\rangle^{k / 2} u_{1}(t, x)\right\|_{L^{\infty} L^{4 d /(2 d-\gamma)}}^{2} \\
\lesssim & \left(M_{k+1}(T)\right)^{3} \varepsilon^{k(1 / 2-\sigma)} T .
\end{aligned}
$$

We observe that

$$
\left\{y \in \mathbf{R}^{d}:\left|y+\eta^{\varepsilon}\right| \leqslant\left|\eta^{\varepsilon}\right|\right\} \subset\left\{y \in \mathbf{R}^{d}:|y| \leqslant 2\left|\eta^{\varepsilon}\right|\right\},
$$

and, for $k>0,\left\langle x-\eta^{\varepsilon}\right\rangle^{k} \lesssim\left\langle x-y-\eta^{\varepsilon}\right\rangle^{k}+|y|^{k}$. Then we have, for all $x \in \mathbf{R}^{d}$,

$$
\begin{aligned}
& \int_{\left\{y:\left|y+\eta^{\varepsilon}\right| \leqslant\left|\eta^{\varepsilon}\right|\right\}}|y|^{-\gamma}\left|u_{1}\left(t, x-y-\eta^{\varepsilon}\right)\right|^{2}\left\langle x-\eta^{\varepsilon}\right\rangle^{k} d y \\
\lesssim & \int_{\mathbf{R}^{d}}|y|^{-\gamma}\left|u_{1}\left(t, x-y-\eta^{\varepsilon}\right)\right|^{2}\left\langle x-y-\eta^{\varepsilon}\right\rangle^{k} d y \\
& +\int_{\left\{y:|y| \leqslant 2\left|\eta^{\varepsilon}\right|\right\}}|y|^{k-\gamma}\left|u_{1}\left(t, x-y-\eta^{\varepsilon}\right)\right|^{2} d y \\
\lesssim & \int_{\mathbf{R}^{d}}|y|^{-\gamma}\left|u_{1}\left(t, x-y-\eta^{\varepsilon}\right)\right|^{2}\left\langle x-y-\eta^{\varepsilon}\right\rangle^{k} d y+\left|\eta^{\varepsilon}\right|^{k-\gamma}\left\|u_{1}(t)\right\|_{L^{2}}^{2},
\end{aligned}
$$

since $k>\gamma$. It follows that

$$
\begin{aligned}
& \int_{[0, T] \backslash I^{\varepsilon}(T)}\left\|\left(\int_{\left|y+\eta^{\varepsilon}\right| \leqslant\left|\eta^{\varepsilon}\right|}|y|^{-\gamma}\left|u_{1}\left(t, x-y-\eta^{\varepsilon}\right)\right|^{2} d y\right) u_{2}(t, x)\right\|_{L^{2}\left(\mathbf{R}^{d}\right)} d t \\
\lesssim & \left(\int_{[0, T] \backslash I^{\varepsilon}(T)} \sup _{\substack{x \in \mathbf{R}^{d} \\
\left|y+\eta^{\varepsilon}\right|>\eta^{\varepsilon} \mid}}\left\langle x-\eta^{\varepsilon}\right\rangle^{-k}\langle x\rangle^{-k} d t\right)\left\|\langle x\rangle^{k} u_{2}(t, x)\right\|_{L^{\infty} L^{2 d /(d-\gamma)}} \\
& \times\left\|\int|y|^{-\gamma}\left|u_{1}\left(t, x-y-\eta^{\varepsilon}\right)\right|^{2}\left\langle x-\eta^{\varepsilon}\right\rangle^{k} d y\right\|_{L^{\infty} L^{2 d / \gamma}} \\
& \lesssim\left(M_{k+1}(T)\right)^{3} \int_{[0, T] \backslash I^{\varepsilon}(T)}\left|\eta^{\varepsilon}\right|^{-k}\left(1+\left|\eta^{\varepsilon}\right|^{k-\gamma}\right) d t \\
& \lesssim\left(M_{k+1}(T)\right)^{3}\left(\varepsilon^{k(1 / 2-\sigma)}+\varepsilon^{\gamma(1 / 2-\sigma)}\right) T \lesssim\left(M_{k+1}(T)\right)^{3} \varepsilon^{\gamma(1 / 2-\sigma)} T,
\end{aligned}
$$


since $k>\gamma$. In $I^{\varepsilon}(T)$, Hölder inequality, Hardy-Littlewood-Sobolev inequality and Sobolev embedding yield similarly

$$
\varepsilon^{\alpha_{c}-1} \int_{I^{\varepsilon}(T)}\left\|N_{I}^{\varepsilon}\right\|_{L^{2}\left(\mathbf{R}^{d}\right)} d t \lesssim\left(M_{1}(T)\right)^{3}\left|I^{\varepsilon}(T)\right|
$$

Note that

$$
\begin{aligned}
\left\|\sqrt{\varepsilon} \nabla \varphi_{j}\right\|_{L^{2}\left(\mathbf{R}^{d}\right)} & \lesssim \sqrt{\varepsilon}\left\|\nabla u_{j}\right\|_{L^{2}\left(\mathbf{R}^{d}\right)}+\left|\xi_{j}\right|\left\|u_{j}\right\|_{L^{2}\left(\mathbf{R}^{d}\right)}, \\
\left\|x \varphi_{j}\right\|_{L^{2}\left(\mathbf{R}^{d}\right)} & \lesssim \sqrt{\varepsilon}\left\|y u_{j}\right\|_{L^{2}\left(\mathbf{R}^{d}\right)}+\left|x_{j}\right|\left\|u_{j}\right\|_{L^{2}\left(\mathbf{R}^{d}\right)} .
\end{aligned}
$$

The $\Sigma_{\varepsilon}$ estimate of $N_{I}^{\varepsilon}$ then follows easily, in view of Lemma 1.3 .

When $T>0$ does not depend on $\varepsilon$, we simply invoke Lemma 6.2 in [6]:

$$
\left|I^{\varepsilon}(T)\right|=\mathcal{O}\left(\varepsilon^{\sigma}\right),
$$

and the proof of the lemma is complete.

6.1. Proof of Theorem 1.9, Due to the lack of natural rescaling for two wave packets, we use a bootstrap argument even on finite time intervals; the operators $A^{\varepsilon}$ and $B^{\varepsilon}$ used in $\$ 4$ are helpful analytically because they have a precise geometrical meaning in terms of one wave packet, and this meaning is lost in the case of two wave packets. Since for $j=1,2$,

$$
\left\|\varphi_{j}^{\varepsilon}(t)\right\|_{L^{r}\left(\mathbf{R}^{d}\right)} \leqslant C(T) \varepsilon^{-\gamma / 8}, \quad \forall t \in[0, T]
$$

the bootstrap argument goes as follows: so long as

$$
\left\|w^{\varepsilon}(t)\right\|_{L^{r}\left(\mathbf{R}^{d}\right)} \leqslant C(T) \varepsilon^{-\gamma / 8},
$$

we estimate the error $w^{\varepsilon}$ with a rather precise rate. Resuming the computations of Proposition 4.2 and using the above bootstrap argument, we have

$$
\left\|w^{\varepsilon}\right\|_{L^{\infty}\left([0, T] ; L^{2}\left(\mathbf{R}^{d}\right)\right)} \lesssim \frac{1}{\varepsilon}\left\|L^{\varepsilon}\right\|_{L^{1}\left([0, T] ; L^{2}\left(\mathbf{R}^{d}\right)\right)}+\frac{1}{\varepsilon}\left\|N_{I}^{\varepsilon}\right\|_{L^{1}\left([0, T] ; L^{2}\left(\mathbf{R}^{d}\right)\right)} .
$$

Similarly, applying $\sqrt{\varepsilon} \nabla$ and $x$ to the equation, resuming an analogue computation as $A^{\varepsilon}$ and $B^{\varepsilon}$, we get

$$
\begin{aligned}
\left\|w^{\varepsilon}\right\|_{L^{\infty}\left([0, T] ; \Sigma_{\varepsilon}\right)} & \lesssim \frac{1}{\varepsilon}\left\|L^{\varepsilon}\right\|_{L^{1}\left([0, T] ; \Sigma_{\varepsilon}\right)}+\frac{1}{\varepsilon}\left\|N_{I}^{\varepsilon}\right\|_{L^{1}\left([0, T] ; \Sigma_{\varepsilon}\right)} \\
& \lesssim \sqrt{\varepsilon}+\varepsilon^{\sigma}+\varepsilon^{\gamma(1 / 2-\sigma)} \lesssim \varepsilon^{\sigma}+\varepsilon^{\gamma(1 / 2-\sigma)},
\end{aligned}
$$

since $0<\sigma<1 / 2$. Optimizing the estimate in $\sigma$, we find

$$
\sigma=\gamma\left(\frac{1}{2}-\sigma\right) \Longleftrightarrow \sigma=\frac{\gamma}{2(1+\gamma)},
$$

which is consistent with $0<\sigma<1 / 2$. Then Gagliardo-Nirenberg inequality yields

$$
\left\|w^{\varepsilon}(t)\right\|_{L^{r}\left(\mathbf{R}^{d}\right)} \lesssim \varepsilon^{-\gamma / 4}\left\|w^{\varepsilon}(t)\right\|_{L^{2}\left(\mathbf{R}^{d}\right)}^{1-\gamma / 4}\left\|\varepsilon \nabla w^{\varepsilon}(t)\right\|_{L^{2}\left(\mathbf{R}^{d}\right)}^{\gamma / 4} \lesssim \varepsilon^{\frac{\gamma}{2(1+\gamma)}-\gamma / 4} .
$$

Now we notice that

$$
\frac{\gamma}{2(1+\gamma)}-\frac{\gamma}{4}>-\frac{\gamma}{8}
$$

so for any $T>0$ independent of $\varepsilon$, we can find $\varepsilon_{0}$ so that (6.3) holds for $t \in[0, T]$ provided that $\left.\varepsilon \in] 0, \varepsilon_{0}\right]$. Theorem $[1.9$ follows. 
6.2. Proof of Theorem 1.10. Since $a_{j} \in \Sigma^{k}$, we have, by Proposition 3.1

$$
M_{k}(t) \leqslant C_{k} e^{C_{k} t} .
$$

From Lemma 6.3 in [6], there exists positive constants $C, C_{1}, C_{2}$ independent of $\varepsilon$ such that

$$
\left|I_{\varepsilon}(t)\right| \leqslant C \varepsilon^{\sigma} e^{C_{1} t}\left|E_{1}-E_{2}\right|^{-2}, \quad \forall t \in\left[0, C_{2} \ln \frac{1}{\varepsilon}\right] .
$$

It follows from Lemma 6.1 that

$$
\varepsilon^{-1}\left\|N_{I}^{\varepsilon}\right\|_{L^{1}\left([0, t] ; \Sigma_{\varepsilon}\right)} \lesssim e^{C t}\left(t \varepsilon^{\gamma(1 / 2-\sigma)}+\varepsilon^{\sigma} e^{C_{1} t}\right) \lesssim e^{C t}\left(\varepsilon^{\gamma(1 / 2-\sigma)}+\varepsilon^{\sigma}\right)
$$

Choosing $k \geqslant 3$ and the same (optimal) $\sigma$ as before,

$$
\sigma=\frac{\gamma}{2(1+\gamma)}
$$

we have

$$
\varepsilon^{-1}\left\|N_{I}^{\varepsilon}\right\|_{L^{1}\left([0, t] ; \Sigma_{\varepsilon}\right)} \lesssim \varepsilon^{\gamma / 2(1+\gamma)} e^{C t} .
$$

Resuming the bootstrap argument and similar arguments as in Section 5 yields Theorem 1.10

\section{REFERENCES}

1. A. Athanassoulis, T. Paul, F. Pezzotti, and M. Pulvirenti, Coherent states propagation for the Hartree equation, Ann. Henri Poincaré (2011), to appear. Archived at http://arxiv.org/abs/1010.4889

2. D. Bambusi, S. Graffi, and T. Paul, Long time semiclassical approximation of quantum flows: a proof of the Ehrenfest time, Asymptot. Anal. 21 (1999), no. 2, 149-160.

3. J. M. Bily and D. Robert, The semi-classical Van Vleck formula. Application to the AharonovBohm effect, Long time behaviour of classical and quantum systems (Bologna, 1999), Ser. Concr. Appl. Math., vol. 1, World Sci. Publ., River Edge, NJ, 2001, pp. 89-106.

4. R. Carles, Semi-classical analysis for nonlinear Schrödinger equations, World Scientific Publishing Co. Pte. Ltd., Hackensack, NJ, 2008.

5. __ Nonlinear Schrödinger equation with time dependent potential, preprint. Archived at http://arxiv.org/abs/0910.4893 2009.

6. R. Carles and C. Fermanian-Kammerer, Nonlinear Coherent States and Ehrenfest Time for Schrödinger Equations, Commun. Math. Phys. 301 (2011), no. 2, 443-472.

7. T. Cazenave, Semilinear Schrödinger equations, Courant Lecture Notes in Mathematics, vol. 10, New York University Courant Institute of Mathematical Sciences, New York, 2003.

8. M. Combescure and D. Robert, Semiclassical spreading of quantum wave packets and applications near unstable fixed points of the classical flow, Asymptot. Anal. 14 (1997), no. 4, 377-404.

9. —_, Quadratic quantum Hamiltonians revisited, Cubo 8 (2006), no. 1, 61-86.

10. _ A phase-space study of the quantum Loschmidt echo in the semiclassical limit, Ann. Henri Poincaré 8 (2007), no. 1, 91-108.

11. D. Fujiwara, A construction of the fundamental solution for the Schrödinger equation, J. Analyse Math. 35 (1979), 41-96.

12. $ـ$ Remarks on the convergence of the Feynman path integrals, Duke Math. J. 47 (1980), no. 3, 559-600.

13. P. Gérard, Oscillations and concentration effects in semilinear dispersive wave equations, J. Funct. Anal. 141 (1996), no. 1, 60-98.

14. J. Ginibre and G. Velo, Scattering theory in the energy space for a class of nonlinear Schrödinger equations, J. Math. Pures Appl. (9) 64 (1985), no. 4, 363-401.

15. G. A. Hagedorn, Semiclassical quantum mechanics. I. The $\hbar \rightarrow 0$ limit for coherent states, Comm. Math. Phys. 71 (1980), no. 1, 77-93.

16. G. A. Hagedorn and A. Joye, Exponentially accurate semiclassical dynamics: propagation, localization, Ehrenfest times, scattering, and more general states, Ann. Henri Poincaré 1 (2000), no. 5, 837-883. 
17. - A time-dependent Born-Oppenheimer approximation with exponentially small error estimates, Comm. Math. Phys. 223 (2001), no. 3, 583-626.

18. T. Kato, On nonlinear Schrödinger equations, Ann. IHP (Phys. Théor.) 46 (1987), no. 1, 113-129.

19. M. Keel and T. Tao, Endpoint Strichartz estimates, Amer. J. Math. 120 (1998), no. 5, 955980.

20. R. G. Littlejohn, The semiclassical evolution of wave packets, Phys. Rep. 138 (1986), no. 4-5, 193-291.

21. T. Paul, Semi-classical methods with emphasis on coherent states, Quasiclassical methods (Minneapolis, MN, 1995), IMA Vol. Math. Appl., vol. 95, Springer, New York, 1997, pp. 5188.

22. D. Robert, On the Herman-Kluk semiclassical approximation, Rev. Math. Phys. 22 (2010), no. $10,1123-1145$.

23. V. Rousse, Semiclassical simple initial value representations, to appear in Ark. för Mat. Archived as http://arxiv.org/abs/0904.0387 2009.

24. T. Swart and V. Rousse, A mathematical justification for the Herman-Kluk propagator, Comm. Math. Phys. 286 (2009), no. 2, 725-750.

25. K. Yajima, Existence of solutions for Schrödinger evolution equations, Comm. Math. Phys. 110 (1987), 415-426.

Department of Mathematical Science, Tsinghua University, Beijing 100084, China

E-mail address: pcao04@gmail.com

Univ. Montpellier 2, Mathématiques, CC 051, F-34095 Montpellier

CNRS, UMR 5149, F-34095 Montpellier, France

E-mail address: Remi.Carles@math.cnrs.fr 